\title{
On aggregation of subcritical Galton-Watson branching processes with regularly varying immigration
}

\author{
Mátyás Barczy ${ }^{\mathrm{a}, 1}$, Fanni K. Nedényi ${ }^{\mathrm{a}, 2}$, and $†$ Gyula Pap $^{\mathrm{b}, 3}$ \\ ${ }^{a}$ MTA-SZTE Analysis and Stochastics Research Group, Bolyai Institute, University of Szeged, \\ Aradi vértanúk tere 1, H-6720 Szeged, Hungary \\ ${ }^{\mathrm{b}}$ Bolyai Institute, University of Szeged, Aradi vértanúk tere 1, H-6720 Szeged, Hungary \\ (e-mail: barczy@math.u-szeged.hu; nfanni@math.u-szeged.hu)
}

Received June 4, 2019; revised April 21, 2020

\begin{abstract}
We study an iterated temporal and contemporaneous aggregation of $N$ independent copies of a strongly stationary subcritical Galton-Watson branching process with regularly varying immigration having index $\alpha \in(0,2)$. We show that limits of finite-dimensional distributions of appropriately centered and scaled aggregated partial-sum processes exist when first taking the limit as $N \rightarrow \infty$ and then the time scale $n \rightarrow \infty$. The limit process is an $\alpha$-stable process if $\alpha \in(0,1) \cup(1,2)$ and a deterministic line with slope 1 if $\alpha=1$.
\end{abstract}

MSC: 60J80, 60F05, 60G10, 60G52, 60G70

Keywords: Galton-Watson branching processes with immigration, temporal and contemporaneous aggregation, multivariate regular variation, stable distribution, limit measure, tail process

\section{Introduction}

The field of temporal and contemporaneous (also called cross-sectional) aggregations of independent stationary stochastic processes is an important and very active research area in the empirical and theoretical statistics and in other areas as well. Robinson [26] and Granger [10] started to investigate the scheme of contemporaneous aggregation of random-coefficient autoregressive processes of order 1 to obtain the long-memory phenomenon in aggregated time series. For surveys on aggregation of different kinds of stochastic processes, see, for example, Pilipauskaite and Surgailis [19], Jirak [13, p. 512], or the arXiv version of Barczy et al. [5].

Recently, Puplinskaite and Surgailis [21,22] studied iterated aggregation of random coefficient autoregressive processes of order 1 with common innovations and so-called idiosyncratic innovations, respectively, belonging to the domain of attraction of an $\alpha$-stable law. Limits of finite-dimensional distributions of appropriately centered and scaled aggregated partial sum processes are shown to exist when first the number of copies $N \rightarrow \infty$ and then the time scale $n \rightarrow \infty$. Very recently, Pilipauskaite et al. [18] extended the results

\footnotetext{
${ }^{1}$ The author is supported by the János Bolyai Research Scholarship of the Hungarian Academy of Sciences.

2 The author is supported by the UNKP-18-3 New National Excellence Program of the Ministry of Human Capacities.

${ }^{3}$ The author was supported by the Ministry for Innovation and Technology, Hungary grant TUDFO/47138-1/2019-ITM.
} 
of Puplinskaitė and Surgailis [22] (idiosyncratic case) deriving limits of finite-dimensional distributions of appropriately centered and scaled aggregated partial-sum processes when first the time scale $n \rightarrow \infty$ and then the number of copies $N \rightarrow \infty$, and when $n \rightarrow \infty$ and $N \rightarrow \infty$ simultaneously with possibly different rates.

The listed references are all about aggregation procedures for times series, mainly for randomized autoregressive processes. According to our knowledge, this question has not been studied before in the literature. In the present paper, we investigate aggregation schemes for some branching processes with low-moment condition. Branching processes, especially Galton-Watson branching processes with immigration, have attracted a lot of attention due to the fact that they are widely used in mathematical biology for modeling the growth of a population in time. In Barczy et al. [3], we started to investigate the limit behavior of temporal and contemporaneous aggregations of independent copies of a stationary multitype Galton-Watson branching process with immigration under third-order moment conditions on the offspring and immigration distributions in the iterated and simultaneous cases as well. In both cases the limit process is a zero-mean Brownian motion with the same covariance function. As of 2020, modeling the COVID-19 contamination of the population of a certain region or country is of great importance. Multitype Galton-Watson processes with immigration have been frequently used to model the spreading of a number of diseases, and they can be applied for this new disease as well. For example, Yanev et al. [29] applied a two-type Galton-Watson process with immigration to model the numbers of detected COVID-19-infected and undetected COVID-19-infected people in a population. The temporal and contemporaneous aggregation of the first coordinate process of the two-type branching process in question would mean the total number of detected infected people up to some given time point across several regions.

In this paper, we study the limit behavior of temporal and contemporaneous aggregations of independent copies of a strongly stationary Galton-Watson branching process $\left(X_{k}\right)_{k \geqslant 0}$ with regularly varying immigration having index in $(0,2)$ (yielding infinite variance) in an iterated idiosyncratic case, namely, when first the number of copies $N \rightarrow \infty$ and then the time scale $n \rightarrow \infty$. Our results are analogous to those of Puplinskaite and Surgailis [22].

The present paper is organized as follows. In Section 2, we first collect our assumptions that are valid for the whole paper, namely, we consider a sequence of independent copies of $\left(X_{k}\right)_{k \geqslant 0}$ such that the expectation of the offspring distribution is less than 1 (so-called subcritical case). In case of $\alpha \in[1,2$ ), we additionally suppose the finiteness of the second moment of the offspring distribution. Under our assumptions, by Basrak et al. [6, Thm. 2.1.1] (see also Theorem D1), the unique stationary distribution of $\left(X_{k}\right)_{k \geqslant 0}$ is also regularly varying with the same index $\alpha$.

In Theorem 1, we show that the appropriately centered and scaled partial-sum process of finite segments of independent copies of $\left(X_{k}\right)_{k \geqslant 0}$ converges to an $\alpha$-stable process. The characteristic function of the $\alpha$-stable limit process is given explicitly as well. The proof of Theorem 1 is based on a slight modification of Theorem 7.1 in Resnick [25], namely, on a result of weak convergence of partial-sum processes toward Lévy processes; see Theorem C1, where we consider a different centering. In the course of the proof of Theorem 1, we need to verify that the so-called limit measures of finite segments of $\left(X_{k}\right)_{k \geqslant 0}$ are in fact Lévy measures. We determine these limit measures explicitly (see part (i) of Proposition D1) applying an expression for the so-called tail measure of a strongly stationary regularly varying sequence based on the corresponding (whole) spectral tail process given by Planinić and Soulier [20, Thm. 3.1].

Whereas the centering in Theorem 1 is the so-called truncated mean, in Corollary 1, we consider noncentering if $\alpha \in(0,1)$ and centering with the mean if $\alpha \in(1,2)$. In both cases the limit process is an $\alpha$-stable process, the same one as in Theorem 1 plus some deterministic drift depending on $\alpha$. Theorem 1 and Corollary 1 together yield the weak convergence of finite-dimensional distributions of appropriately centered and scaled contemporaneous aggregations of independent copies of $\left(X_{k}\right)_{k \geqslant 0}$ toward the corresponding finitedimensional distributions of a strongly stationary subcritical autoregressive process of order 1 with $\alpha$-stable innovations as the number of copies tends to infinity; see Corollary 2 and Proposition 1.

Theorem 2 contains our main result: we determine the weak limit of appropriately centered and scaled finite-dimensional distributions of temporal and contemporaneous aggregations of independent copies of $\left(X_{k}\right)_{k \geqslant 0}$, where the limit is taken so that first the number of copies tends to infinity and then the time corresponding to temporal aggregation tends to infinity. It turns out that the limit process is an $\alpha$-stable process if $\alpha \in(0,1) \cup(1,2)$ and a deterministic line with slope 1 if $\alpha=1$. We consider different kinds of centerings, 
and we give the explicit characteristic function of the limit process as well. In Remark 2, we rewrite this characteristic function in case of $\alpha \in(0,1)$ in terms of the spectral tail process of $\left(X_{k}\right)_{k \geqslant 0}$.

We close the paper with four appendices. Appendix A is devoted to some properties of the underlying punctured space $\mathbb{R}^{d} \backslash\{\mathbf{0}\}$ and vague convergence. In Appendix B, we recall the notion of a regularly varying random vector and its limit measure, and in Proposition D1 the limit measure of an appropriate positively homogeneous real-valued function of a regularly varying random vector. In Appendix C, we formulate a result on weak convergence of partial-sum processes toward Lévy processes by slightly modifying Theorem 7.1 in Resnick [25] with a different centering. In the end, we recall a result on the tail behavior and forward tail process of $\left(X_{k}\right)_{k \geqslant 0}$ due to Basrak et al. [6], and we determine the limit measures of finite segments of $\left(X_{k}\right)_{k \geqslant 0}$; see Appendix D.

Finally, we summarize the novelties of the paper. According to our knowledge, studying aggregation of regularly varying Galton-Watson branching processes with immigration has not been considered before. In the proofs, we make use of the explicit form of the (whole) spectral tail process and a very recent result of Planinić and Soulier [20, Thm. 3.1] about the tail measure of strongly stationary sequences. We explicitly determine the limit measures of finite segments of $\left(X_{k}\right)_{k \geqslant 0}$; see part (i) of Proposition D1.

For brevity of the paper, we omit some (simple) proofs and calculation steps. However, all these details are included in our arXiv version Barczy et al. [4] of this paper.

In a companion paper, we will study another iterated idiosyncratic aggregation scheme, namely, when first the time scale $n \rightarrow \infty$ and then the number of copies $N \rightarrow \infty$.

\section{Main results}

Let $\mathbb{Z}_{+}, \mathbb{N}, \mathbb{Q}, \mathbb{R}, \mathbb{R}_{+}, \mathbb{R}_{++}, \mathbb{R}_{-}, \mathbb{R}_{--}$, and $\mathbb{C}$ denote the set of nonnegative integers, positive integers, rational numbers, real numbers, nonnegative real numbers, positive real numbers, nonpositive real numbers, negative real numbers and complex numbers, respectively. For each $d \in \mathbb{N}$, the natural basis in $\mathbb{R}^{d}$ is denoted by $\boldsymbol{e}_{1}, \ldots, \boldsymbol{e}_{d}$. Put $\mathbf{1}_{d}:=(1, \ldots, 1)^{\top}$ and $\mathbb{S}^{d-1}:=\left\{\boldsymbol{x} \in \mathbb{R}^{d}:\|\boldsymbol{x}\|=1\right\}$, where $\|\boldsymbol{x}\|$ denotes the Euclidean norm of $\boldsymbol{x} \in \mathbb{R}^{d}$, and denote by $\mathcal{B}\left(\mathbb{S}^{d-1}\right)$ the Borel $\sigma$-field of $\mathbb{S}^{d-1}$. For a probability measure $\mu$ on $\mathbb{R}^{d}, \widehat{\mu}$ denotes its characteristic function, that is, $\widehat{\mu}(\boldsymbol{\theta}):=\int_{\mathbb{R}^{d}} \mathrm{e}^{\mathrm{i}\langle\boldsymbol{\theta}, \boldsymbol{x}\rangle} \mu(\mathrm{d} \boldsymbol{x})$ for $\boldsymbol{\theta} \in \mathbb{R}^{d}$. Convergence in distribution and almost sure convergence of random variables and weak convergence of probability measures is denoted by $\stackrel{\mathcal{D}}{\rightarrow}, \stackrel{\text { a.s. }}{\rightarrow}$, and $\stackrel{w}{\rightarrow}$, respectively. Equality in distribution is denoted by $\stackrel{\mathcal{D}}{=}$. We use $\stackrel{\mathcal{D}_{\mathrm{f}}}{\rightarrow}$ or $\mathcal{D}_{\mathrm{f}}$-lim for weak convergence of finite-dimensional distributions. A function $f: \mathbb{R}_{+} \rightarrow \mathbb{R}^{d}$ is called càdlàg if it is right continuous with left limits. Let $\mathbb{D}\left(\mathbb{R}_{+}, \mathbb{R}^{d}\right)$ denote the space of all $\mathbb{R}^{d}$-valued càdlàg functions on $\mathbb{R}_{+}$. Let $\mathcal{B}\left(\mathbb{D}\left(\mathbb{R}_{+}, \mathbb{R}^{d}\right)\right)$ denote the Borel $\sigma$-algebra on $\mathbb{D}\left(\mathbb{R}_{+}, \mathbb{R}^{d}\right)$ for the metric defined by Jacod and Shiryaev [11, Chap. VI, (1.26)]. With this metric, $\mathbb{D}\left(\mathbb{R}_{+}, \mathbb{R}^{d}\right)$ is a complete separable metric space, and the topology induced by this metric is the socalled Skorokhod topology. For $\mathbb{R}^{d}$-valued stochastic processes $\left(\mathcal{Y}_{t}\right)_{t \in \mathbb{R}_{+}}$and $\left(\mathcal{Y}_{t}^{(n)}\right)_{t \in \mathbb{R}_{+}}, n \in \mathbb{N}$, with càdlàg paths, we write $\mathcal{Y}^{(n)} \stackrel{\mathcal{D}}{\longrightarrow} \mathcal{Y}$ as $n \rightarrow \infty$ if the distribution of $\mathcal{Y}^{(n)}$ on the space $\left(\mathbb{D}\left(\mathbb{R}_{+}, \mathbb{R}^{d}\right), \mathcal{B}\left(\mathbb{D}\left(\mathbb{R}_{+}, \mathbb{R}^{d}\right)\right)\right)$ converges weakly to the distribution of $\mathcal{Y}$ on the space $\left(\mathbb{D}\left(\mathbb{R}_{+}, \mathbb{R}^{d}\right), \mathcal{B}\left(\mathbb{D}\left(\mathbb{R}_{+}, \mathbb{R}^{d}\right)\right)\right)$ as $n \rightarrow \infty$.

Let $\left(X_{k}\right)_{k \in \mathbb{Z}_{+}}$be a Galton-Watson branching process with immigration. For $k, j \in \mathbb{Z}_{+}$, we denote the number of individuals in the $k$ th generation by $X_{k}$, the number of offsprings produced by the $j$ th individual belonging to the $(k-1)$ th generation by $\xi_{k, j}$, and the number of immigrants in the $k$ th generation by $\varepsilon_{k}$. Then we have

$$
X_{k}=\sum_{j=1}^{X_{k-1}} \xi_{k, j}+\varepsilon_{k}, \quad k \in \mathbb{N},
$$

where $\sum_{j=1}^{0}:=0$. Here $\left\{X_{0}, \xi_{k, j}, \varepsilon_{k}: k, j \in \mathbb{N}\right\}$ are supposed to be independent nonnegative integervalued random variables. Moreover, $\left\{\xi_{k, j}: k, j \in \mathbb{N}\right\}$ and $\left\{\varepsilon_{k}: k \in \mathbb{N}\right\}$ are supposed to consist of identically distributed random variables, respectively. For notational convenience, let $\xi$ and $\varepsilon$ be independent random variables such that $\xi \stackrel{\mathcal{D}}{=} \xi_{1,1}$ and $\varepsilon \stackrel{\mathcal{D}}{=} \varepsilon_{1}$. 
If $m_{\xi}:=\mathbf{E}(\xi) \in[0,1)$ and $\sum_{\ell=1}^{\infty} \log (\ell) \mathbf{P}(\varepsilon=\ell)<\infty$, then the Markov chain $\left(X_{k}\right)_{k \in \mathbb{Z}_{+}}$admits a unique stationary distribution $\pi$; see, for example, Quine [23]. Note that if $m_{\xi} \in[0,1)$ and $\mathbf{P}(\varepsilon=0)=1$, then $\sum_{\ell=1}^{\infty} \log (\ell) \mathbf{P}(\varepsilon=\ell)=0$, and $\pi$ is the Dirac measure $\delta_{0}$ concentrated at the point 0 . In fact, $\pi=\delta_{0}$ if and only if $\mathbf{P}(\varepsilon=0)=1$. Moreover, if $m_{\xi}=0$ (which is equivalent to $\mathbf{P}(\xi=0)=1$ ), then $\pi$ is the distribution of $\varepsilon$.

In what follows, we formulate our assumptions valid for the whole paper. We assume that $m_{\xi} \in[0,1)$ (so-called subcritical case) and $\varepsilon$ is regularly varying with index $\alpha \in(0,2)$, that is, $\mathbf{P}(\varepsilon>x) \in \mathbb{R}_{++}$for all $x \in \mathbb{R}_{++}$, and

$$
\lim _{x \rightarrow \infty} \frac{\mathbf{P}(\varepsilon>q x)}{\mathbf{P}(\varepsilon>x)}=q^{-\alpha} \quad \text { for all } q \in \mathbb{R}_{++}
$$

Then $\mathbf{P}(\varepsilon=0)<1$ and $\sum_{\ell=1}^{\infty} \log (\ell) \mathbf{P}(\varepsilon=\ell)<\infty$ (see, e.g., Barczy et al. [2, Lemma E.5]), and hence the Markov process $\left(X_{k}\right)_{k \in \mathbb{Z}_{+}}$admits a unique stationary distribution $\pi$. We suppose that $X_{0} \stackrel{\mathcal{D}}{=} \pi$, yielding that the Markov chain $\left(X_{k}\right)_{k \in \mathbb{Z}_{+}}$is strongly stationary. In case of $\alpha \in[1,2)$, we additionally suppose that $\mathbf{E}\left(\xi^{2}\right)<\infty$. By Basrak et al. [6, Thm. 2.1.1] (see also Theorem D1) $X_{0}$ is regularly varying with index $\alpha$, yielding the existence of a sequence $\left(a_{N}\right)_{N \in \mathbb{N}}$ in $\mathbb{R}_{++}$with $N \mathbf{P}\left(X_{0}>a_{N}\right) \rightarrow 1$ as $N \rightarrow \infty$; see, for example, Lemma B2. Let us fix an arbitrary sequence $\left(a_{N}\right)_{N \in \mathbb{N}}$ in $\mathbb{R}_{++}$with this property. In fact, $a_{N}=N^{1 / \alpha} L(N)$, $N \in \mathbb{N}$, for some slowly varying continuous function $L: \mathbb{R}_{++} \rightarrow \mathbb{R}_{++}$; see, e.g., Araujo and Giné [1, p. 90, Exercise 6]. Let $X^{(j)}=\left(X_{k}^{(j)}\right)_{k \in \mathbb{Z}_{+}}, j \in \mathbb{N}$, be a sequence of independent copies of $\left(X_{k}\right)_{k \in \mathbb{Z}_{+}}$. We mention that we consider so-called idiosyncratic immigrations, that is, the immigrations $\left(\varepsilon_{k}^{(j)}\right)_{k \in \mathbb{N}}, j \in \mathbb{N}$, belonging to $\left(X_{k}^{(j)}\right)_{k \in \mathbb{Z}_{+}}, j \in \mathbb{N}$, are independent. One could study the case of common immigrations as well, that is, when $\left(\varepsilon_{k}^{(j)}\right)_{k \in \mathbb{N}}=\left(\varepsilon_{k}^{(1)}\right)_{k \in \mathbb{N}}, j \in \mathbb{N}$.

Theorem 1. For each $k \in \mathbb{Z}_{+}$,

$$
\left(\frac{1}{a_{N}} \sum_{j=1}^{\lfloor N t\rfloor}\left(X_{0}^{(j)}-\mathbf{E}\left(X_{0}^{(j)} \mathbf{1}_{\left\{X_{0}^{(j)} \leqslant a_{N}\right\}}\right), \ldots, X_{k}^{(j)}-\mathbf{E}\left(X_{k}^{(j)} \mathbf{1}_{\left\{X_{k}^{(j)} \leqslant a_{N}\right\}}\right)\right)^{\top}\right)_{t \in \mathbb{R}_{+}} \stackrel{\mathcal{D}}{\longrightarrow}\left(\mathcal{X}_{t}^{(k, \alpha)}\right)_{t \in \mathbb{R}_{+}}
$$

as $N \rightarrow \infty$, where $\left(\mathcal{X}_{t}^{(k, \alpha)}\right)_{t \in \mathbb{R}_{+}}$is a $(k+1)$-dimensional $\alpha$-stable process such that the characteristic function of the distribution $\mu_{k, \alpha}$ of $\mathcal{X}_{1}^{(k, \alpha)}$ has the form

$$
\begin{aligned}
& \widehat{\mu_{k, \alpha}}(\boldsymbol{\theta}) \\
& =\exp \left\{\left(1-m_{\xi}^{\alpha}\right) \sum_{j=0}^{k} \int_{0}^{\infty}\left(\mathrm{e}^{\mathrm{i}\left\langle\boldsymbol{\theta}, \boldsymbol{v}_{j}^{(k)}\right\rangle u}-1-\mathrm{i} u \sum_{\ell=j+1}^{k+1}\left\langle\boldsymbol{e}_{\ell}, \boldsymbol{\theta}\right\rangle\left\langle\boldsymbol{e}_{\ell}, \boldsymbol{v}_{j}^{(k)}\right\rangle \mathbf{1}_{(0,1]}\left(u\left\langle\boldsymbol{e}_{\ell}, \boldsymbol{v}_{j}^{(k)}\right\rangle\right)\right) \alpha u^{-1-\alpha} \mathrm{d} u\right\}
\end{aligned}
$$

for $\boldsymbol{\theta} \in \mathbb{R}^{k+1}$ with the $(k+1)$-dimensional vectors

$$
\boldsymbol{v}_{0}^{(k)}:=\left(1-m_{\xi}^{\alpha}\right)^{-1 / \alpha}\left[\begin{array}{c}
1 \\
m_{\xi} \\
m_{\xi}^{2} \\
\vdots \\
m_{\xi}^{k}
\end{array}\right], \quad \boldsymbol{v}_{1}^{(k)}:=\left[\begin{array}{c}
0 \\
1 \\
m_{\xi} \\
\vdots \\
m_{\xi}^{k-1}
\end{array}\right], \quad \boldsymbol{v}_{2}^{(k)}:=\left[\begin{array}{c}
0 \\
0 \\
1 \\
\vdots \\
m_{\xi}^{k-2}
\end{array}\right], \quad \ldots, \quad \boldsymbol{v}_{k}^{(k)}:=\left[\begin{array}{c}
0 \\
0 \\
\vdots \\
0 \\
1
\end{array}\right]
$$


Moreover, for $\boldsymbol{\theta} \in \mathbb{R}^{k+1}$,

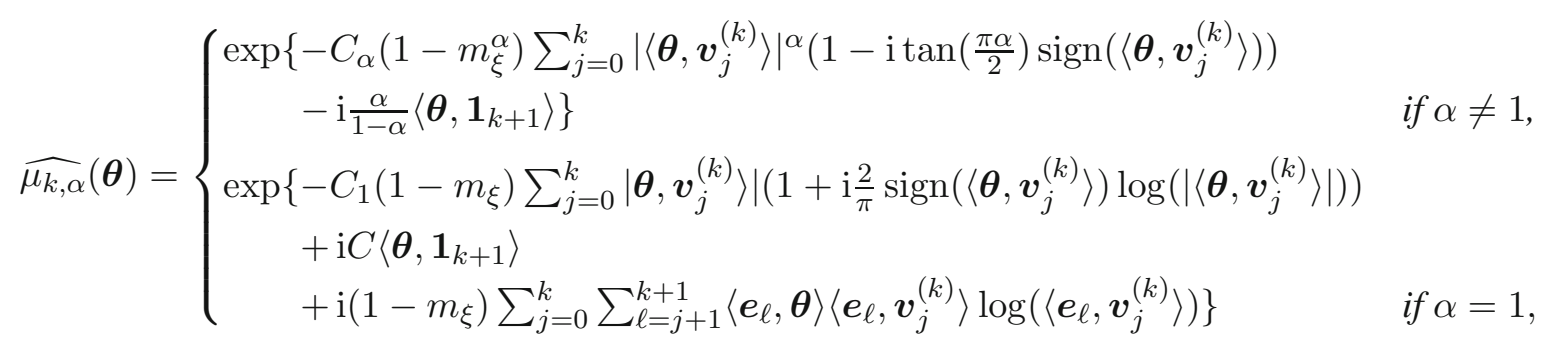

with $0 \log (0):=0$,

$$
C_{\alpha}:= \begin{cases}\frac{\Gamma(2-\alpha)}{1-\alpha} \cos \left(\frac{\pi \alpha}{2}\right) & \text { if } \alpha \neq 1 \\ \frac{\pi}{2} & \text { if } \alpha=1\end{cases}
$$

and

$$
C:=\int_{1}^{\infty} u^{-2} \sin u \mathrm{~d} u+\int_{0}^{1} u^{-2}(\sin u-u) \mathrm{d} u
$$

The next remark is devoted to some properties of $\mu_{k, \alpha}$.

Remark 1. By the proof of Theorem 1 (see (3.4)) it turns out that the Lévy measure of $\mu_{k, \alpha}$ is

$$
\nu_{k, \alpha}(B)=\left(1-m_{\xi}^{\alpha}\right) \sum_{j=0}^{k}\left\|\boldsymbol{v}_{j}^{(k)}\right\|^{\alpha} \int_{0}^{\infty} \mathbf{1}_{B}\left(u \frac{\boldsymbol{v}_{j}^{(k)}}{\left\|\boldsymbol{v}_{j}^{(k)}\right\|}\right) \alpha u^{-\alpha-1} \mathrm{~d} u, \quad B \in \mathcal{B}\left(\mathbb{R}_{0}^{k+1}\right)
$$

where the space $\mathbb{R}_{0}^{k+1}:=\mathbb{R}^{k+1} \backslash\{\mathbf{0}\}$ and its topological properties are discussed in Appendix A. The radial part of $\nu_{k, \alpha}$ is $u^{-\alpha-1} \mathrm{~d} u$, and the spherical part of $\nu_{k, \alpha}$ is any positive constant multiple of the measure $\sum_{j=0}^{k}\left\|\boldsymbol{v}_{j}^{(k)}\right\|{ }^{\alpha} \epsilon_{\boldsymbol{v}_{j}^{(k)} /\left\|\boldsymbol{v}_{j}^{(k)}\right\|}$ on $\mathbb{S}^{k}$, where for any $\boldsymbol{x} \in \mathbb{R}^{k+1}, \epsilon_{\boldsymbol{x}}$ denotes the Dirac measure concentrated at the point $\boldsymbol{x}$. Particularly, the support of $\nu_{k, \alpha}$ is $\cup_{j=0}^{k}\left(\mathbb{R}_{++} \boldsymbol{v}_{j}^{(k)}\right)$. The vectors $\boldsymbol{v}_{0}^{(k)}, \ldots, \boldsymbol{v}_{k}^{(k)}$ form a basis in $\mathbb{R}^{k+1}$, and hence there is no proper linear subspace $V$ of $\mathbb{R}^{k+1}$ covering the support of $\nu_{k, \alpha}$. Consequently, $\mu_{k, \alpha}$ is a nondegenerate measure in the sense that there are no $\boldsymbol{a} \in \mathbb{R}^{k+1}$ and a proper linear subspace $V$ of $\mathbb{R}^{k+1}$ such that $a+V$ covers the support of $\mu_{k, \alpha}$; see, for example, Sato [27, Prop. 24.17(ii)].

The centering in Theorem 1 can be simplified in case of $\alpha \neq 1$. Namely, if $\alpha \in(0,1]$, then for each $t \in \mathbb{R}_{++}$, by Lemma B3,

$$
\begin{aligned}
\frac{\lfloor N t\rfloor}{a_{N}} \mathbf{E}\left(X_{0} \mathbf{1}_{\left\{X_{0} \leqslant a_{N}\right\}}\right) & =\frac{\lfloor N t\rfloor}{N} \frac{\mathbf{E}\left(X_{0} \mathbf{1}_{\left\{X_{0} \leqslant a_{N}\right\}}\right)}{a_{N} \mathbf{P}\left(X_{0}>a_{N}\right)} N \mathbf{P}\left(X_{0}>a_{N}\right) \\
& \rightarrow\left\{\begin{array}{ll}
\frac{\alpha}{1-\alpha} t & \text { for } \alpha \in(0,1), \\
\infty & \text { for } \alpha=1
\end{array} \text { as } N \rightarrow \infty .\right.
\end{aligned}
$$

In a similar way, if $\alpha \in(1,2)$, then for each $t \in \mathbb{R}_{++}$,

$$
\frac{\lfloor N t\rfloor}{a_{N}} \mathbf{E}\left(X_{0} \mathbf{1}_{\left\{X_{0} \leqslant a_{N}\right\}}\right)=\frac{\lfloor N t\rfloor}{a_{N}} \mathbf{E}\left(X_{0}\right)-\frac{\lfloor N t\rfloor}{a_{N}} \mathbf{E}\left(X_{0} \mathbf{1}_{\left\{X_{0}>a_{N}\right\}}\right),
$$


where $\lim _{N \rightarrow \infty}\lfloor N t\rfloor / a_{N}=\lim _{N \rightarrow \infty} t N^{1-1 / \alpha} L(N)^{-1}=\infty$, and, by Lemma B3,

$$
\frac{\lfloor N t\rfloor}{a_{N}} \mathbf{E}\left(X_{0} \mathbf{1}_{\left\{X_{0}>a_{N}\right\}}\right) \rightarrow \frac{\alpha}{\alpha-1} t \quad \text { as } N \rightarrow \infty .
$$

This shows that in case of $\alpha \in(0,1)$, there is no need for centering; in case of $\alpha \in(1,2)$, we can center with the expectation as well, whereas in case of $\alpha=1$, neither noncentering nor centering with the expectation works even if the expectation does exist. More precisely, without centering in case of $\alpha \in(0,1)$ or with centering with the expectation in case of $\alpha \in(1,2)$, we have the following convergences.

Corollary 1. In case of $\alpha \in(0,1)$, for each $k \in \mathbb{Z}_{+}$, we have

$$
\left(\frac{1}{a_{N}} \sum_{j=1}^{\lfloor N t\rfloor}\left(X_{0}^{(j)}, \ldots, X_{k}^{(j)}\right)^{\top}\right)_{t \in \mathbb{R}_{+}} \stackrel{\mathcal{D}}{\longrightarrow}\left(\boldsymbol{\mathcal { X }}_{t}^{(k, \alpha)}+\frac{\alpha}{1-\alpha} t \mathbf{1}_{k+1}\right)_{t \in \mathbb{R}_{+}} \quad \text { as } N \rightarrow \infty
$$

and, in case of $\alpha \in(1,2)$, for each $k \in \mathbb{Z}_{+}$, we have

$$
\begin{aligned}
& \left(\frac{1}{a_{N}} \sum_{j=1}^{\lfloor N t\rfloor}\left(X_{0}^{(j)}-\mathbf{E}\left(X_{0}^{(j)}\right), \ldots, X_{k}^{(j)}-\mathbf{E}\left(X_{k}^{(j)}\right)\right)^{\top}\right)_{t \in \mathbb{R}_{+}} \\
& \stackrel{\mathcal{D}}{\longrightarrow}\left(\mathcal{X}_{t}^{(k, \alpha)}+\frac{\alpha}{1-\alpha} t \mathbf{1}_{k+1}\right)_{t \in \mathbb{R}_{+}} \text {as } N \rightarrow \infty .
\end{aligned}
$$

Moreover, $\left(\mathcal{X}_{t}^{(k, \alpha)}+(\alpha /(1-\alpha)) t \mathbf{1}_{k+1}\right)_{t \in \mathbb{R}_{+}}$is a $(k+1)$-dimensional $\alpha$-stable process such that the characteristic function of $\mathcal{X}_{1}^{(k, \alpha)}+(\alpha /(1-\alpha)) \mathbf{1}_{k+1}$ has the form

$$
\begin{aligned}
& \mathbf{E}\left(\exp \left\{\mathrm{i}\left\langle\boldsymbol{\theta}, \boldsymbol{\mathcal { X }}_{1}^{(k, \alpha)}+\frac{\alpha}{1-\alpha} \mathbf{1}_{k+1}\right\rangle\right\}\right) \\
& = \begin{cases}\exp \left\{\left(1-m_{\xi}^{\alpha}\right) \sum_{j=0}^{k} \int_{0}^{\infty}\left(\mathrm{e}^{\mathrm{i}\left\langle\boldsymbol{\theta}, \boldsymbol{v}_{j}^{(k)}\right\rangle u}-1\right) \alpha u^{-1-\alpha} \mathrm{d} u\right\} & \text { if } \alpha \in(0,1), \\
\exp \left\{\left(1-m_{\xi}^{\alpha}\right) \sum_{j=0}^{k} \int_{0}^{\infty}\left(\mathrm{e}^{\mathrm{i}\left\langle\boldsymbol{\theta}, \boldsymbol{v}_{j}^{(k)}\right\rangle u}-1-\mathrm{i}\left\langle\boldsymbol{\theta}, \boldsymbol{v}_{j}^{(k)}\right\rangle u\right) \alpha u^{-1-\alpha} \mathrm{d} u\right\} & \text { if } \alpha \in(1,2),\end{cases} \\
& =\exp \left\{-C_{\alpha}\left(1-m_{\xi}^{\alpha}\right) \sum_{j=0}^{k}\left|\left\langle\boldsymbol{\theta}, \boldsymbol{v}_{j}^{(k)}\right\rangle\right|^{\alpha}\left(1-\mathrm{i} \tan \left(\frac{\pi \alpha}{2}\right) \operatorname{sign}\left(\left\langle\boldsymbol{\theta}, \boldsymbol{v}_{j}^{(k)}\right\rangle\right)\right)\right\} \quad \text { if } \alpha \neq 1
\end{aligned}
$$

for $\boldsymbol{\theta} \in \mathbb{R}^{k+1}$.

Let $\left(\mathcal{Y}_{k}^{(\alpha)}\right)_{k \in \mathbb{Z}_{+}}$be a strongly stationary process such that

$$
\left(\mathcal{Y}_{k}^{(\alpha)}\right)_{k \in\{0, \ldots, K\}} \stackrel{\mathcal{D}}{=} \mathcal{X}_{1}^{(K, \alpha)} \quad \text { for each } K \in \mathbb{Z}_{+}
$$

The existence of $\left(\mathcal{Y}_{k}^{(\alpha)}\right)_{k \in \mathbb{Z}_{+}}$follows from the Kolmogorov extension theorem. Its strong stationarity is a consequence of Theorem 1 together with the strong stationarity of $\left(X_{k}\right)_{k \in \mathbb{Z}_{+}}$. We note that the common distribution 
of $\mathcal{Y}_{k}^{(\alpha)}, k \in \mathbb{Z}_{+}$, depends only on $\alpha$; it does not depend on $m_{\xi}$, since its characteristic function has the form

$$
\begin{aligned}
& \mathbf{E}\left(\mathrm{e}^{\mathrm{i} \vartheta \mathcal{Y}_{0}^{(\alpha)}}\right) \\
& =\mathbf{E}\left(\mathrm{e}^{\mathrm{i} \vartheta \boldsymbol{X}_{1}^{(0, \alpha)}}\right) \\
& =\exp \left\{\left(1-m_{\xi}^{\alpha}\right) \int_{0}^{\infty}\left(\mathrm{e}^{\mathrm{i} \vartheta\left(1-m_{\xi}^{\alpha}\right)^{-1 / \alpha} u}-1-\mathrm{i} u \vartheta\left(1-m_{\xi}^{\alpha}\right)^{-1 / \alpha} \mathbf{1}_{(0,1]}\left(u\left(1-m_{\xi}^{\alpha}\right)^{-1 / \alpha}\right)\right) \alpha u^{-1-\alpha} \mathrm{d} u\right\} \\
& \quad=\exp \left\{\int_{0}^{\infty}\left(\mathrm{e}^{\mathrm{i} \vartheta v}-1-\mathrm{i} \vartheta v \mathbf{1}_{(0,1]}(v)\right) \alpha v^{-1-\alpha} \mathrm{d} v\right\}, \quad \vartheta \in \mathbb{R} .
\end{aligned}
$$

Proposition 1. For each $\alpha \in(0,2)$, the strongly stationary process $\left(\mathcal{Y}_{k}^{(\alpha)}\right)_{k \in \mathbb{Z}_{+}}$is a subcritical autoregressive process of order 1 with autoregressive coefficient $m_{\xi}$ and $\alpha$-stable innovations, namely,

where

$$
\mathcal{Y}_{k}^{(\alpha)}=m_{\xi} \mathcal{Y}_{k-1}^{(\alpha)}+\widetilde{\varepsilon}_{k}^{(\alpha)}, \quad k \in \mathbb{N},
$$

$$
\widetilde{\varepsilon}_{k}^{(\alpha)}:=\mathcal{Y}_{k}^{(\alpha)}-m_{\xi} \mathcal{Y}_{k-1}^{(\alpha)}, \quad k \in \mathbb{N}
$$

is a sequence of independent identically distributed $\alpha$-stable random variables such that for all $k \in \mathbb{N}, \widetilde{\varepsilon}_{k}^{(\alpha)}$ is independent of $\left(\mathcal{Y}_{0}^{(\alpha)}, \ldots, \mathcal{Y}_{k-1}^{(\alpha)}\right)^{\top}$. Therefore $\left(\mathcal{Y}_{k}^{(\alpha)}\right)_{k \in \mathbb{Z}_{+}}$is a strongly stationary time-homogeneous Markov process.

Theorem 1 and Corollary 1 have the following consequences for a contemporaneous aggregation of independent copies with different centerings.

\section{Corollary 2.}

(i) For each $\alpha \in(0,2)$,

$$
\left(\frac{1}{a_{N}} \sum_{j=1}^{N}\left(X_{k}^{(j)}-\mathbf{E}\left(X_{k}^{(j)} \mathbf{1}_{\left\{X_{k}^{(j)} \leqslant a_{N}\right\}}\right)\right)\right)_{k \in \mathbb{Z}_{+}} \stackrel{\mathcal{D}_{\mathrm{f}}}{\longrightarrow}\left(\mathcal{Y}_{k}^{(\alpha)}\right)_{k \in \mathbb{Z}_{+}} \text {as } N \rightarrow \infty,
$$

(ii) in case of $\alpha \in(0,1)$,

$$
\left(\frac{1}{a_{N}} \sum_{j=1}^{N} X_{k}^{(j)}\right)_{k \in \mathbb{Z}_{+}} \stackrel{\mathcal{D}_{\mathrm{f}}}{\longrightarrow}\left(\mathcal{Y}_{k}^{(\alpha)}+\frac{\alpha}{1-\alpha}\right)_{k \in \mathbb{Z}_{+}} \text {as } N \rightarrow \infty,
$$

(iii) in case of $\alpha \in(1,2)$,

$$
\left(\frac{1}{a_{N}} \sum_{j=1}^{N}\left(X_{k}^{(j)}-\mathbf{E}\left(X_{k}^{(j)}\right)\right)\right)_{k \in \mathbb{Z}_{+}} \stackrel{\mathcal{D}_{\mathrm{f}}}{\longrightarrow}\left(\mathcal{Y}_{k}^{(\alpha)}+\frac{\alpha}{1-\alpha}\right)_{k \in \mathbb{Z}_{+}} \text {as } N \rightarrow \infty,
$$

where $\left(\mathcal{Y}^{(k)}\right)_{k \in \mathbb{Z}_{+}}$is given by (2.2).

We will present limit theorems for the aggregated stochastic process $\left(\sum_{k=1}^{\lfloor n t\rfloor} \sum_{j=1}^{N} X_{k}^{(j)}\right)_{t \in \mathbb{R}_{+}}$with different centerings and scalings and in an iterated manner such that first $N$ and then $n$ converge to infinity. 
Theorem 2. In case of $\alpha \in(0,1)$, we have

$$
\underset{n \rightarrow \infty}{\mathcal{D}_{\mathrm{f}}-\lim _{N \rightarrow \infty}} \mathcal{D}_{\mathrm{f}}-\lim \left(\frac{1}{n^{1 / \alpha} a_{N}} \sum_{k=1}^{\lfloor n t\rfloor} \sum_{j=1}^{N}\left(X_{k}^{(j)}-\mathbf{E}\left(X_{k}^{(j)} \mathbf{1}_{\left\{X_{k}^{(j)} \leqslant a_{N}\right\}}\right)\right)\right)_{t \in \mathbb{R}_{+}}=\left(\mathcal{Z}_{t}^{(\alpha)}+\frac{\alpha}{1-\alpha} t\right)_{t \in \mathbb{R}_{+}}
$$

and

$$
\underset{n \rightarrow \infty}{\mathcal{D}_{\mathrm{f}}-\lim _{N \rightarrow \infty}} \mathcal{D}_{\mathrm{f}-\lim }\left(\frac{1}{n^{1 / \alpha} a_{N}} \sum_{k=1}^{\lfloor n t\rfloor} \sum_{j=1}^{N} X_{k}^{(j)}\right)_{t \in \mathbb{R}_{+}}=\left(\mathcal{Z}_{t}^{(\alpha)}+\frac{\alpha}{1-\alpha} t\right)_{t \in \mathbb{R}_{+}}
$$

in case of $\alpha=1$, we have

$$
\underset{n \rightarrow \infty}{\mathcal{D}_{\mathrm{f}}-\lim } \underset{\mathcal{D}_{\mathrm{f}}-\lim }{ }\left(\frac{1}{n \log (n) a_{N}} \sum_{k=1}^{\lfloor n t\rfloor} \sum_{j=1}^{N}\left(X_{k}^{(j)}-\mathbf{E}\left(X_{k}^{(j)} \mathbf{1}_{\left\{X_{k}^{(j)} \leqslant a_{N}\right\}}\right)\right)\right)_{t \in \mathbb{R}_{+}}=(t)_{t \in \mathbb{R}_{+}}
$$

and in case of $\alpha \in(1,2)$, we have

$$
\underset{n \rightarrow \infty}{\mathcal{D}_{\mathrm{f}}-\lim _{N \rightarrow \infty}} \mathcal{D}_{\mathrm{f}-\lim }\left(\frac{1}{n^{1 / \alpha} a_{N}} \sum_{k=1}^{\lfloor n t\rfloor} \sum_{j=1}^{N}\left(X_{k}^{(j)}-\mathbf{E}\left(X_{k}^{(j)}\right)\right)\right)_{t \in \mathbb{R}_{+}}=\left(\mathcal{Z}_{t}^{(\alpha)}+\frac{\alpha}{1-\alpha} t\right)_{t \in \mathbb{R}_{+}},
$$

where $\left(\mathcal{Z}_{t}^{(\alpha)}\right)_{t \in \mathbb{R}_{+}}$is an $\alpha$-stable process such that the characteristic function of the distribution of $\mathcal{Z}_{1}^{(\alpha)}$ has the form

$$
\mathbf{E}\left(\mathrm{e}^{\mathrm{i} \vartheta \mathcal{Z}_{1}^{(\alpha)}}\right)=\exp \left\{\mathrm{i} b_{\alpha} \vartheta+\frac{1-m_{\xi}^{\alpha}}{\left(1-m_{\xi}\right)^{\alpha}} \int_{0}^{\infty}\left(\mathrm{e}^{\mathrm{i} \vartheta u}-1-\mathrm{i} \vartheta u \mathbf{1}_{(0,1]}(u)\right) \alpha u^{-1-\alpha} \mathrm{d} u\right\}, \quad \vartheta \in \mathbb{R},
$$

where

$$
b_{\alpha}:=\left(\frac{1-m_{\xi}^{\alpha}}{\left(1-m_{\xi}\right)^{\alpha}}-1\right) \frac{\alpha}{1-\alpha}, \quad \alpha \in(0,1) \cup(1,2),
$$

and $\left(\mathcal{Z}_{t}^{(\alpha)}+(\alpha /(1-\alpha)) t\right)_{t \in \mathbb{R}_{+}}$is an $\alpha$-stable process such that the characteristic function of the distribution of $\mathcal{Z}_{1}^{(\alpha)}+\alpha /(1-\alpha)$ has the form

$$
\begin{aligned}
& \mathbf{E}\left(\exp \left\{\mathrm{i} \vartheta\left(\mathcal{Z}_{1}^{(\alpha)}+\frac{\alpha}{1-\alpha}\right)\right\}\right) \\
& = \begin{cases}\exp \left\{\frac{1-m_{\xi}^{\alpha}}{\left(1-m_{\xi}\right)^{\alpha}} \int_{0}^{\infty}\left(\mathrm{e}^{\mathrm{i} \vartheta u}-1\right) \alpha u^{-1-\alpha} \mathrm{d} u\right\} & \text { if } \alpha \in(0,1), \\
\exp \left\{\frac{1-m_{\xi}^{\alpha}}{\left(1-m_{\xi}\right)^{\alpha}} \int_{0}^{\infty}\left(\mathrm{e}^{\mathrm{i} \vartheta u}-1-\mathrm{i} \vartheta u\right) \alpha u^{-1-\alpha} \mathrm{d} u\right\} & \text { if } \alpha \in(1,2), \\
\quad=\exp \left\{-C_{\alpha} \frac{1-m_{\xi}^{\alpha}}{\left(1-m_{\xi}\right)^{\alpha}}|\vartheta|^{\alpha}\left(1-\mathrm{i} \tan \left(\frac{\pi \alpha}{2}\right) \operatorname{sign}(\vartheta)\right)\right\} & \text { if } \alpha \in(0,1) \cup(1,2)\end{cases}
\end{aligned}
$$

for $\vartheta \in \mathbb{R}$. 
Remark 2. Note that in accordance with Basrak and Segers [7, Remark 4.8] and Mikosch and Wintenberger [17, p. 171], in case of $\alpha \in(0,1)$, we have

$$
\begin{aligned}
& \mathbf{E}\left(\exp \left\{\mathrm{i} \vartheta\left(\mathcal{Z}_{1}^{(\alpha)}+\frac{\alpha}{1-\alpha}\right)\right\}\right) \\
& \quad=\exp \left\{-\int_{0}^{\infty} \mathbf{E}\left[\exp \left(\mathrm{i} u \vartheta \sum_{\ell=1}^{\infty} \Theta_{\ell}\right)-\exp \left(\mathrm{i} u \vartheta \sum_{\ell=0}^{\infty} \Theta_{\ell}\right)\right] \alpha u^{-\alpha-1} \mathrm{~d} u\right\}
\end{aligned}
$$

for $\vartheta \in \mathbb{R}$, where $\left(\Theta_{\ell}\right)_{\ell \in \mathbb{Z}_{+}}$is the (forward) spectral tail process of $\left(X_{\ell}\right)_{\ell \in \mathbb{Z}_{+}}$given in (3.7) and (3.8). We also remark that (2.7) does not hold in case of $\alpha \in(1,2)$, which is somewhat unexpected in view of Mikosch and Wintenberger [17, p. 171].

\section{Proofs}

Proof of Theorem 1. Let $k \in \mathbb{Z}_{+}$. We are going to apply Theorem C1 with $d=k+1$ and $\boldsymbol{X}_{N, j}:=$ $a_{N}^{-1}\left(X_{0}^{(j)}, \ldots, X_{k}^{(j)}\right)^{\top}, N, j \in \mathbb{N}$. The aim of the following discussion is checking condition (C.1) of Theorem C1, namely

$$
N \mathbf{P}\left(\boldsymbol{X}_{N, 1} \in \cdot\right)=N \mathbf{P}\left(a_{N}^{-1}\left(X_{0}^{(1)}, \ldots, X_{k}^{(1)}\right)^{\top} \in \cdot\right) \stackrel{v}{\longrightarrow} \nu_{k, \alpha}(\cdot) \quad \text { on } \mathbb{R}_{0}^{k+1} \text { as } N \rightarrow \infty,
$$

where $\nu_{k, \alpha}$ is a Lévy measure on $\mathbb{R}_{0}^{k+1}$. For all $N \in \mathbb{N}$ and $B \in \mathcal{B}\left(\mathbb{R}_{0}^{k+1}\right)$, we can write

$$
N \mathbf{P}\left(\boldsymbol{X}_{N, 1} \in B\right)=N \mathbf{P}\left(X_{0}>a_{N}\right) \frac{\mathbf{P}\left(a_{N}^{-1}\left(X_{0}, \ldots, X_{k}\right)^{\top} \in B\right)}{\mathbf{P}\left(X_{0}>a_{N}\right)} .
$$

By the assumption we have $N \mathbf{P}\left(X_{0}>a_{N}\right) \rightarrow 1$ as $N \rightarrow \infty$, yielding also $a_{N} \rightarrow \infty$ as $N \rightarrow \infty$, and, consequently, it suffices to show that

$$
\frac{\mathbf{P}\left(x^{-1}\left(X_{0}, \ldots, X_{k}\right)^{\top} \in \cdot\right)}{\mathbf{P}\left(X_{0}>x\right)} \stackrel{v}{\longrightarrow} \nu_{k, \alpha}(\cdot) \quad \text { on } \mathbb{R}_{0}^{k+1} \text { as } x \rightarrow \infty,
$$

where $\nu_{k, \alpha}$ is a Lévy measure on $\mathbb{R}_{0}^{k+1}$. In fact, by Theorem $\mathrm{D} 2,\left(X_{0}, \ldots, X_{k}\right)^{\top}$ is regularly varying with index $\alpha$, and hence by Proposition B1 we know that

$$
\frac{\mathbf{P}\left(x^{-1}\left(X_{0}, \ldots, X_{k}\right)^{\top} \in \cdot\right)}{\mathbf{P}\left(\left\|\left(X_{0}, \ldots, X_{k}\right)^{\top}\right\|>x\right)} \stackrel{v}{\longrightarrow} \widetilde{\nu}_{k, \alpha}(\cdot) \quad \text { on } \mathbb{R}_{0}^{k+1} \text { as } x \rightarrow \infty,
$$

where $\widetilde{\nu}_{k, \alpha}$ is the so-called limit measure of $\left(X_{0}, \ldots, X_{k}\right)^{\top}$. Applying Proposition D1 for the canonical projection $p_{0}: \mathbb{R}^{k+1} \rightarrow \mathbb{R}$ given by $p_{0}(\boldsymbol{x}):=x_{0}$ for $\boldsymbol{x}=\left(x_{0}, \ldots, x_{k}\right)^{\top} \in \mathbb{R}^{k+1}$, which is continuous and positively homogeneous of degree 1 , we obtain

$$
\frac{\mathbf{P}\left(X_{0}>x\right)}{\mathbf{P}\left(\left\|\left(X_{0}, \ldots, X_{k}\right)^{\top}\right\|>x\right)} \rightarrow \widetilde{\nu}_{k, \alpha}\left(T_{1}\right) \quad \text { as } x \rightarrow \infty
$$

with $T_{1}:=\left\{\boldsymbol{x} \in \mathbb{R}_{0}^{k+1}: p_{0}(\boldsymbol{x})>1\right\}$, where we have $\widetilde{\nu}_{k, \alpha}\left(T_{1}\right) \in(0,1]$. Indeed,

$$
\mathbf{P}\left(X_{0}>x\right) \leqslant \mathbf{P}\left(\left\|\left(X_{0}, \ldots, X_{k}\right)^{\top}\right\|>x\right),
$$


and hence $\widetilde{\nu}_{k, \alpha}\left(T_{1}\right) \leqslant 1$. Moreover, by the strong stationarity of $\left(X_{k}\right)_{k \in \mathbb{Z}_{+}}$we have

$$
\mathbf{P}\left(\left\|\left(X_{0}, \ldots, X_{k}\right)^{\top}\right\|>x\right) \leqslant \sum_{j=0}^{k} \mathbf{P}\left(X_{j}>\frac{x}{\sqrt{k+1}}\right)=(k+1) \mathbf{P}\left(X_{0}>\frac{x}{\sqrt{k+1}}\right),
$$

and thus

$$
\frac{\mathbf{P}\left(X_{0}>x\right)}{\mathbf{P}\left(\left\|\left(X_{0}, \ldots, X_{k}\right)^{\top}\right\|>x\right)} \geqslant \frac{\mathbf{P}\left(X_{0}>x\right)}{(k+1) \mathbf{P}\left(X_{0}>\frac{x}{\sqrt{k+1}}\right)} \rightarrow(k+1)^{-1-\alpha / 2} \quad \text { as } x \rightarrow \infty,
$$

since $X_{0}$ is regularly varying with index $\alpha$, and hence $\widetilde{\nu}_{k, \alpha}\left(T_{1}\right) \in(0,1]$, as desired. Consequently, (3.2) holds with $\nu_{k, \alpha}=\widetilde{\nu}_{k, \alpha} / \widetilde{\nu}_{k, \alpha}\left(T_{1}\right)$. In general, we do not know whether $\nu_{k, \alpha}$ is a Lévy measure on $\mathbb{R}_{0}^{k+1}$ or not. So, additional work is needed. We will determine $\nu_{k, \alpha}$ explicitly using a result of Planinić and Soulier [20].

The aim of the following discussion is applying Theorem 3.1 in Planinić and Soulier [20] to determine $\nu_{k, \alpha}$, namely, we will prove that for each Borel-measurable function $f: \mathbb{R}_{0}^{k+1} \rightarrow \mathbb{R}_{+}$,

$$
\int_{\mathbb{R}_{0}^{k+1}} f(\boldsymbol{x}) \nu_{k, \alpha}(\mathrm{d} \boldsymbol{x})=\left(1-m_{\xi}^{\alpha}\right) \sum_{j=0}^{k} \int_{0}^{\infty} f\left(u \boldsymbol{v}_{j}^{(k)}\right) \alpha u^{-\alpha-1} \mathrm{~d} u .
$$

Let $\left(X_{\ell}\right)_{\ell \in \mathbb{Z}}$ be a strongly stationary extension of $\left(X_{\ell}\right)_{\ell \in \mathbb{Z}_{+}}$. For all $i, j \in \mathbb{Z}$ with $i \leqslant j$, by Theorem D2, $\left(X_{i}, \ldots, X_{j}\right)^{\top}$ is regularly varying with index $\alpha$, and hence by the strong stationarity of $\left(X_{k}\right)_{k \in \mathbb{Z}}$ and the previous discussion we know that

$$
\frac{\mathbf{P}\left(x^{-1}\left(X_{i}, \ldots, X_{j}\right)^{\top} \in \cdot\right)}{\mathbf{P}\left(X_{0}>x\right)}=\frac{\mathbf{P}\left(x^{-1}\left(X_{0}, \ldots, X_{j-i}\right)^{\top} \in \cdot\right)}{\mathbf{P}\left(X_{0}>x\right)} \stackrel{v}{\longrightarrow} \nu_{i, j, \alpha}(\cdot) \quad \text { on } \mathbb{R}_{0}^{j-i+1} \text { as } x \rightarrow \infty,
$$

where $\nu_{i, j, \alpha}:=\nu_{j-i, \alpha}$ is a nonzero locally finite measure on $\mathbb{R}_{0}^{j-i+1}$. According to Basrak and Segers [7, Thm. 2.1], there exists a sequence $\left(Y_{\ell}\right)_{\ell \in \mathbb{Z}}$ of random variables, called the (whole) tail process of $\left(X_{\ell}\right)_{\ell \in \mathbb{Z}}$, such that

$$
\mathbf{P}\left(x^{-1}\left(X_{i}, \ldots, X_{j}\right)^{\top} \in \cdot \mid X_{0}>x\right) \stackrel{w}{\longrightarrow} \mathbf{P}\left(\left(Y_{i}, \ldots, Y_{j}\right)^{\top} \in \cdot\right) \quad \text { as } x \rightarrow \infty .
$$

Let $K$ be a random variable with geometric distribution

$$
\mathbf{P}(K=k)=m_{\xi}^{\alpha k}\left(1-m_{\xi}^{\alpha}\right), \quad k \in \mathbb{Z}_{+} .
$$

Especially, if $m_{\xi}=0$, then $\mathbf{P}(K=0)=1$. If $m_{\xi} \in(0,1)$, then we have

$$
Y_{\ell}= \begin{cases}m_{\xi}^{\ell} Y_{0} & \text { if } \ell \geqslant 0 \\ m_{\xi}^{\ell} Y_{0} \mathbf{1}_{\{K \geqslant-\ell\}} & \text { if } \ell<0\end{cases}
$$

where $Y_{0}$ is a random variable independent of $K$ with Pareto distribution

$$
\mathbf{P}\left(Y_{0}>y\right)= \begin{cases}y^{-\alpha} & \text { if } y \in[1, \infty) \\ 1 & \text { if } y \in(-\infty, 1)\end{cases}
$$

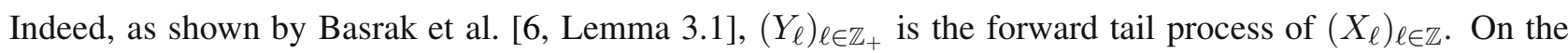
other hand, by Janssen and Segers [12, Ex. 6.2], $\left(Y_{\ell}\right)_{\ell \in \mathbb{Z}}$ is the tail process of the stationary solution $\left(X_{\ell}^{\prime}\right)_{\ell \in \mathbb{Z}}$ 
to the stochastic recurrence equation $X_{\ell}^{\prime}=\mu_{A} X_{\ell-1}^{\prime}+B_{\ell}, \ell \in \mathbb{Z}$. Since the distribution of the forward tail process determines the distribution of the (whole) tail process (see Basrak and Segers [7, Thm. 3.1(ii)]), it follows that $\left(Y_{\ell}\right)_{\ell \in \mathbb{Z}}$ represents the tail process of $\left(X_{\ell}\right)_{\ell \in \mathbb{Z}}$. If $m_{\xi}=0$, then we can easily check that

$$
Y_{\ell}= \begin{cases}Y_{0} & \text { if } \ell=0 \\ 0 & \text { if } \ell \neq 0\end{cases}
$$

By (3.5) and (3.6) we have $Y_{\ell} \stackrel{\text { a.s. }}{\rightarrow} 0$ as $\ell \rightarrow \infty$ or $\ell \rightarrow-\infty$, and hence condition (3.1) in Planinić and Soulier [20] is satisfied.

Moreover, there exists a unique measure $\nu_{\alpha}$ on $\mathbb{R}^{\mathbb{Z}}$ endowed with the cylindrical $\sigma$-algebra $\mathcal{B}(\mathbb{R})^{\otimes \mathbb{Z}}$ such that $\nu_{\alpha}(\{\mathbf{0}\})=0$ and for all $i, j \in \mathbb{Z}$ with $i \leqslant j$, we have $\nu_{\alpha} \circ p_{i, j}^{-1}=\nu_{i, j, \alpha}$ on $\mathbb{R}_{0}^{j-i+1}$, where $p_{i, j}$ denotes the canonical projection $p_{i, j}: \mathbb{R}^{\mathbb{Z}} \rightarrow \mathbb{R}^{j-i+1}$ given by $p_{i, j}(\boldsymbol{y}):=\left(y_{i}, \ldots, y_{j}\right)$ for $\boldsymbol{y}=\left(y_{\ell}\right)_{\ell \in \mathbb{Z}} \in \mathbb{R}^{\mathbb{Z}}$; see, for example, Planinić and Soulier [20]. The measure $\nu_{\alpha}$ is called the tail measure of $\left(X_{\ell}\right)_{\ell \in \mathbb{Z}}$.

If $m_{\xi} \in(0,1)$, then by (3.5) the (whole) spectral tail process $\Theta=\left(\Theta_{\ell}\right)_{\ell \in \mathbb{Z}}$ of $\left(X_{\ell}\right)_{\ell \in \mathbb{Z}}$ is given by

$$
\Theta_{\ell}:=\frac{Y_{\ell}}{\left|Y_{0}\right|}= \begin{cases}m_{\xi}^{\ell} & \text { if } \ell \geqslant 0, \\ m_{\xi}^{\ell} \mathbf{1}_{\{K \geqslant-\ell\}} & \text { if } \ell<0 .\end{cases}
$$

If $m_{\xi}=0$, then by (3.6)

$$
\Theta_{\ell}:=\frac{Y_{\ell}}{\left|Y_{0}\right|}= \begin{cases}1 & \text { if } \ell=0 \\ 0 & \text { if } \ell \neq 0\end{cases}
$$

Let us introduce the so-called infargmax functional $I: \mathbb{R}^{\mathbb{Z}} \rightarrow \mathbb{Z} \cup\{-\infty, \infty\}$. For $\boldsymbol{y}=\left(y_{\ell}\right)_{\ell \in \mathbb{Z}} \in \mathbb{R}^{\mathbb{Z}}$, the value $I(\boldsymbol{y})$ is the first time when the supremum $\sup _{\ell \in \mathbb{Z}}\left|y_{\ell}\right|$ is achieved; more precisely,

$$
I(\boldsymbol{y}):= \begin{cases}\ell \in \mathbb{Z} & \text { if } \sup _{m \leqslant \ell-1}\left|y_{m}\right|<\left|y_{\ell}\right| \text { and } \sup _{m \geqslant \ell+1}\left|y_{m}\right| \leqslant\left|y_{\ell}\right|, \\ -\infty & \text { if } \sup _{m \leqslant \ell}\left|y_{m}\right|=\sup _{m \in \mathbb{Z}}\left|y_{m}\right| \text { for all } \ell \in \mathbb{Z}, \\ \infty & \text { if } \sup _{m \leqslant \ell}\left|y_{m}\right|<\sup _{m \in \mathbb{Z}}\left|y_{m}\right| \text { for all } \ell \in \mathbb{Z} .\end{cases}
$$

We have $\mathbf{P}(I(\boldsymbol{\Theta})=-K)=1$, and hence the condition $\mathbf{P}(I(\boldsymbol{\Theta}) \in \mathbb{Z})=1$ of Theorem 3.1 in Planinić and Soulier [20] is satisfied.

Consequently, we may apply Theorem 3.1 in Planinić and Soulier [20] for the nonnegative measurable function $H: \mathbb{R}^{\mathbb{Z}} \rightarrow \mathbb{R}_{+}$given by $H(\boldsymbol{y})=f \circ p_{0, k}$, where $f: \mathbb{R}^{k+1} \rightarrow \mathbb{R}_{+}$is a measurable function with $f(\mathbf{0})=0$. By (3.2) in Planinić and Soulier [20] we obtain

$$
\begin{aligned}
\int_{\mathbb{R}_{0}^{k+1}} f(\boldsymbol{x}) \nu_{k, \alpha}(\mathrm{d} \boldsymbol{x}) & =\int_{\mathbb{R}^{k+1}} f(\boldsymbol{x}) \nu_{0, k, \alpha}(\mathrm{d} \boldsymbol{x})=\int_{\mathbb{R}^{k+1}} f(\boldsymbol{x})\left(\nu_{\alpha} \circ p_{0, k}^{-1}\right)(\mathrm{d} \boldsymbol{x}) \\
& =\int_{\mathbb{R}^{\mathbb{Z}}} f\left(p_{0, k}(\boldsymbol{y})\right) \nu_{\alpha}(\mathrm{d} \boldsymbol{y})=\int_{\mathbb{R}^{\mathbb{Z}}} H(\boldsymbol{y}) \nu_{\alpha}(\mathrm{d} \boldsymbol{y}) \\
& =\sum_{\ell \in \mathbb{Z}} \int_{0}^{\infty} \mathbf{E}\left(H\left(u L^{\ell}(\boldsymbol{\Theta})\right) \mathbf{1}_{\{I(\boldsymbol{\Theta})=0\}}\right) \alpha u^{-\alpha-1} \mathrm{~d} u,
\end{aligned}
$$


where $L$ denotes the backshift operator $L: \mathbb{R}^{\mathbb{Z}} \rightarrow \mathbb{R}^{\mathbb{Z}}$ given by $L(\boldsymbol{y})=\left(L(\boldsymbol{y})_{k}\right)_{k \in \mathbb{Z}}:=\left(y_{k-1}\right)_{k \in \mathbb{Z}}$ for $\boldsymbol{y}=\left(y_{k}\right)_{k \in \mathbb{Z}} \in \mathbb{R}^{\mathbb{Z}}$. Using $\mathbf{P}(I(\boldsymbol{\Theta})=-K)=1$, we obtain

$$
\int_{\mathbb{R}_{0}^{k+1}} f(\boldsymbol{x}) \nu_{k, \alpha}(\mathrm{d} \boldsymbol{x})=\sum_{\ell \in \mathbb{Z}} \int_{0}^{\infty} \mathbf{E}\left(f\left(p_{0, k}\left(u L^{\ell}(\boldsymbol{\Theta})\right)\right) \mathbf{1}_{\{K=0\}}\right) \alpha u^{-\alpha-1} \mathrm{~d} u .
$$

For all $k \in \mathbb{Z}_{+}$and $u \in \mathbb{R}_{+}$, on the event $\{K=0\}$, by (3.7) and (3.8) we have

$$
p_{0, k}\left(u L^{\ell}(\boldsymbol{\Theta})\right)= \begin{cases}\mathbf{0} \in \mathbb{R}^{k+1} & \text { if } \ell>k, \\ u \boldsymbol{v}_{\ell}^{(k)} & \text { if } \ell \in\{1, \ldots, k\}, \\ \left(1-m_{\xi}^{\alpha}\right)^{1 / \alpha} m_{\xi}^{-\ell} u \boldsymbol{v}_{0}^{(k)} & \text { if } \ell \leqslant 0,\end{cases}
$$

and hence, using $\mathbf{P}(K=0)=1-m_{\xi}^{\alpha}$, we obtain

$$
\begin{aligned}
\int_{\mathbb{R}_{0}^{k+1}} f(\boldsymbol{x}) \nu_{k, \alpha}(\mathrm{d} \boldsymbol{x})= & \left(1-m_{\xi}^{\alpha}\right) \sum_{\ell \leqslant 0} \int_{0}^{\infty} f\left(\left(1-m_{\xi}^{\alpha}\right)^{1 / \alpha} m_{\xi}^{-\ell} u \boldsymbol{v}_{0}^{(k)}\right) \alpha u^{-\alpha-1} \mathrm{~d} u \\
& +\left(1-m_{\xi}^{\alpha}\right) \sum_{\ell=1}^{k} \int_{0}^{\infty} f\left(u \boldsymbol{v}_{\ell}^{(k)}\right) \alpha u^{-\alpha-1} \mathrm{~d} u \\
= & \left(1-m_{\xi}^{\alpha}\right) \sum_{\ell=0}^{k} \int_{0}^{\infty} f\left(u \boldsymbol{v}_{\ell}^{(k)}\right) \alpha u^{-\alpha-1} \mathrm{~d} u .
\end{aligned}
$$

The measure $\nu_{k, \alpha}$ is a Lévy measure on $\mathbb{R}_{0}^{k+1}$, since (3.4) implies

$$
\begin{aligned}
\int_{\mathbb{R}_{0}^{k+1}} \min \left\{1,\|\boldsymbol{x}\|^{2}\right\} \nu_{k, \alpha}(\mathrm{d} \boldsymbol{x}) & =\left(1-m_{\xi}^{\alpha}\right) \sum_{j=0}^{k} \int_{0}^{\infty} \min \left\{1,\left\|u \boldsymbol{v}_{j}^{(k)}\right\|^{2}\right\} \alpha u^{-\alpha-1} \mathrm{~d} u \\
& =\left(1-m_{\xi}^{\alpha}\right) \sum_{j=0}^{k}\left\|\boldsymbol{v}_{j}^{(k)}\right\|^{\alpha} \int_{0}^{\infty} \min \left\{1, w^{2}\right\} \alpha w^{-\alpha-1} \mathrm{~d} w \\
& =\frac{2\left(1-m_{\xi}^{\alpha}\right)}{2-\alpha} \sum_{j=0}^{k}\left\|\boldsymbol{v}_{j}^{(k)}\right\|^{\alpha}<\infty .
\end{aligned}
$$

Consequently, we obtain (3.2) and hence (3.1), so condition (C.1) is satisfied.

The aim of the following discussion is checking condition (C.2) of Theorem C1, namely,

$$
\lim _{\varepsilon \downarrow 0} \limsup _{N \rightarrow \infty} N \mathbf{E}\left(a_{N}^{-2}\left(X_{\ell}^{(j)}\right)^{2} \mathbf{1}_{\left\{X_{\ell}^{(j)} \leqslant a_{N} \varepsilon\right\}}\right)=\lim _{\varepsilon \downarrow 0} \limsup _{N \rightarrow \infty} N \mathbf{E}\left(a_{N}^{-2} X_{0}^{2} \mathbf{1}_{\left\{X_{0} \leqslant a_{N} \varepsilon\right\}}\right)=0
$$

for all $j \in \mathbb{N}$ and $\ell \in\{0, \ldots, k\}$. By Lemma B3 with $\beta=2$ we have

$$
\lim _{x \rightarrow \infty} \frac{x^{2} \mathbf{P}\left(X_{0}>x\right)}{\mathbf{E}\left(X_{0}^{2} \mathbf{1}_{\left\{X_{0} \leqslant x\right\}}\right)}=\frac{2-\alpha}{\alpha}
$$


and hence for all $\varepsilon \in \mathbb{R}_{++}$, using again that $X_{0}$ is regularly varying with index $\alpha$, we have

$$
\begin{aligned}
N \mathbf{E}\left(a_{N}^{-2} X_{0}^{2} \mathbf{1}_{\left\{X_{0} \leqslant a_{N} \varepsilon\right\}}\right) & =\frac{\mathbf{E}\left(X_{0}^{2} \mathbf{1}_{\left\{X_{0} \leqslant a_{N} \varepsilon\right\}}\right)}{\left(a_{N} \varepsilon\right)^{2} \mathbf{P}\left(X_{0}>a_{N} \varepsilon\right)} \frac{\mathbf{P}\left(X_{0}>a_{N} \varepsilon\right)}{\mathbf{P}\left(X_{0}>a_{N}\right)} \varepsilon^{2} N \mathbf{P}\left(X_{0}>a_{N}\right) \\
& \rightarrow \frac{\alpha}{2-\alpha} \varepsilon^{2-\alpha}
\end{aligned}
$$

as $N \rightarrow \infty$, and taking the limit as $\varepsilon \downarrow 0$, we conclude (3.9).

Consequently, we may apply Theorem $\mathrm{C} 1$, and we obtain the desired convergence, where $\left(\mathcal{X}_{t}^{(k, \alpha)}\right)_{t \in \mathbb{R}_{+}}$is an $\alpha$-stable process such that the characteristic function of the distribution $\mu_{k, \alpha}$ of $\boldsymbol{\mathcal { X }}_{1}^{(k, \alpha)}$ has the form given in Theorem 1. Indeed, (3.4) is valid for each Borel-measurable function $f: \mathbb{R}_{0}^{k+1} \rightarrow \mathbb{C}$ as well, for which the real and imaginary parts of the right-hand side of (3.4) are well defined. Hence for all $\boldsymbol{\theta} \in \mathbb{R}^{k+1}$, by (C.3),

$$
\begin{aligned}
& \widehat{\mu_{k, \alpha}}(\boldsymbol{\theta}) \\
& =\exp \left\{\int_{\mathbb{R}_{0}^{k+1}}\left(\mathrm{e}^{\mathrm{i}\langle\boldsymbol{\theta}, \boldsymbol{y}\rangle}-1-\mathrm{i} \sum_{\ell=1}^{k+1}\left\langle\boldsymbol{e}_{\ell}, \boldsymbol{\theta}\right\rangle\left\langle\boldsymbol{e}_{\ell}, \boldsymbol{y}\right\rangle \mathbf{1}_{(0,1]}\left(\left|\left\langle\boldsymbol{e}_{\ell}, \boldsymbol{y}\right\rangle\right|\right)\right) \nu_{k, \alpha}(\mathrm{d} \boldsymbol{y})\right\} \\
& =\exp \left\{\left(1-m_{\xi}^{\alpha}\right) \sum_{j=0}^{k} \int_{0}^{\infty}\left(\mathrm{e}^{\mathrm{i}\left\langle\boldsymbol{\theta}, \boldsymbol{v}_{j}^{(k)}\right\rangle u}-1-\mathrm{i} u \sum_{\ell=j+1}^{k+1}\left\langle\boldsymbol{e}_{\ell}, \boldsymbol{\theta}\right\rangle\left\langle\boldsymbol{e}_{\ell}, \boldsymbol{v}_{j}^{(k)}\right\rangle \mathbf{1}_{(0,1]}\left(u\left\langle\boldsymbol{e}_{\ell}, \boldsymbol{v}_{j}^{(k)}\right\rangle\right)\right) \alpha u^{-1-\alpha} \mathrm{d} u\right\},
\end{aligned}
$$

since the real and imaginary parts of the exponent in the last expression are well defined. The rest is a standard calculation as we can see in our arXiv preprint Barczy et al. [4].

Proof of Corollary 1. It follows by the continuous mapping theorem; the reader can find the details in our arXiv preprint Barczy et al. [4].

Proof of Proposition 1. It is a consequence of Theorem 1 and (2.2); for a detailed proof, see our arXiv preprint Barczy et al. [4].

Proof of Corollary 2. It follows from Theorem 1 and Corollary 1 using the continuous mapping theorem.

Proof of Theorem 2. In case of $\alpha \in(0,1)$, by (2.1) with $t=1$ we have

$$
\lim _{N \rightarrow \infty} \frac{\lfloor n t\rfloor N}{n^{1 / \alpha} a_{N}} \mathbf{E}\left(X_{0} \mathbf{1}_{\left\{X_{0} \leqslant a_{N}\right\}}\right)=\frac{\lfloor n t\rfloor}{n^{1 / \alpha}} \frac{\alpha}{1-\alpha} \rightarrow 0 \quad \text { as } n \rightarrow \infty,
$$

and hence, by Slutsky's lemma, (2.3) is a consequence of (2.4).

For each $n \in \mathbb{N}$, by Corollary 2 and the continuous mapping theorem we obtain

$$
\left(\frac{1}{n^{1 / \alpha} a_{N}} \sum_{k=1}^{\lfloor n t\rfloor} \sum_{j=1}^{N} X_{k}^{(j)}\right)_{t \in \mathbb{R}_{+}} \stackrel{\mathcal{D}_{\mathrm{f}}}{\longrightarrow}\left(\frac{1}{n^{1 / \alpha}} \sum_{k=1}^{\lfloor n t\rfloor}\left(\mathcal{Y}_{k}^{(\alpha)}+\frac{\alpha}{1-\alpha}\right)\right)_{t \in \mathbb{R}_{+}} \quad \text { as } N \rightarrow \infty
$$

in case of $\alpha \in(0,1)$ and

$$
\left(\frac{1}{n^{1 / \alpha} a_{N}} \sum_{k=1}^{\lfloor n t\rfloor} \sum_{j=1}^{N}\left(X_{k}^{(j)}-\mathbf{E}\left(X_{k}^{(j)}\right)\right)\right)_{t \in \mathbb{R}_{+}} \stackrel{\mathcal{D}_{\mathrm{f}}}{\longrightarrow}\left(\frac{1}{n^{1 / \alpha}} \sum_{k=1}^{\lfloor n t\rfloor}\left(\mathcal{Y}_{k}^{(\alpha)}+\frac{\alpha}{1-\alpha}\right)\right)_{t \in \mathbb{R}_{+}} \quad \text { as } N \rightarrow \infty
$$


in case of $\alpha \in(1,2)$. Consequently, to prove (2.4) and (2.6), we need to show that for each $\alpha \in(0,1) \cup(1,2)$, we have

$$
\left(\frac{1}{n^{1 / \alpha}} \sum_{k=1}^{\lfloor n t\rfloor}\left(\mathcal{Y}_{k}^{(\alpha)}+\frac{\alpha}{1-\alpha}\right)\right)_{t \in \mathbb{R}_{+}} \stackrel{\mathcal{D}_{\mathrm{f}}}{\longrightarrow}\left(\mathcal{Z}_{t}^{(\alpha)}+\frac{\alpha}{1-\alpha} t\right)_{t \in \mathbb{R}_{+}} \quad \text { as } n \rightarrow \infty .
$$

For all $\alpha \in(0,1) \cup(1,2), n \in \mathbb{N}, d \in \mathbb{N}, t_{1}, \ldots, t_{d} \in \mathbb{R}_{++}$with $t_{1}<\cdots<t_{d}$ and $\vartheta_{1}, \ldots, \vartheta_{d} \in \mathbb{R}$, we have

$$
\begin{aligned}
& \mathbf{E}\left(\exp \left\{\mathrm{i} \sum_{\ell=1}^{d} \frac{\vartheta_{\ell}}{n^{1 / \alpha}} \sum_{k=\left\lfloor n t_{\ell-1}\right\rfloor+1}^{\left\lfloor n t_{\ell}\right\rfloor}\left(\mathcal{Y}_{k}^{(\alpha)}+\frac{\alpha}{1-\alpha}\right)\right\}\right) \\
& =\mathbf{E}\left(\exp \left\{\mathrm{i}\left\langle n^{-1 / \alpha} \boldsymbol{\theta}_{n}, \boldsymbol{\mathcal { X }}_{1}^{\left(\left\lfloor n t_{d}\right\rfloor, \alpha\right)}+\frac{\alpha}{1-\alpha} \mathbf{1}_{\left\lfloor n t_{d}\right\rfloor+1}\right\rangle\right\}\right)
\end{aligned}
$$

with $t_{0}:=0$ and

$$
\boldsymbol{\theta}_{n}:=\sum_{\ell=1}^{d} \vartheta_{\ell} \sum_{k=\left\lfloor n t_{\ell-1}\right\rfloor+1}^{\left\lfloor n t_{\ell}\right\rfloor} \boldsymbol{e}_{k+1} \in \mathbb{R}^{\left\lfloor n t_{d}\right\rfloor+1}
$$

For each $\alpha \in(0,1) \cup(1,2)$, by the explicit form of the characteristic function of $\boldsymbol{\mathcal { X }}_{1}^{\left(\left\lfloor n t_{d}\right\rfloor, \alpha\right)}$ given in Theorem 1 we have

$$
\begin{aligned}
& \mathbf{E}\left(\exp \left\{\mathrm{i}\left\langle n^{-1 / \alpha} \boldsymbol{\theta}_{n}, \boldsymbol{\mathcal { X }}_{1}^{\left(\left\lfloor n t_{d}\right\rfloor, \alpha\right)}+\frac{\alpha}{1-\alpha} \mathbf{1}_{\left\lfloor n t_{d}\right\rfloor+1}\right\rangle\right\}\right) \\
& \quad=\exp \left\{-C_{\alpha}\left(1-m_{\xi}^{\alpha}\right) \sum_{j=0}^{\left\lfloor n t_{d}\right\rfloor}\left|\left\langle n^{-1 / \alpha} \boldsymbol{\theta}_{n}, \boldsymbol{v}_{j}^{\left(\left\lfloor n t_{d}\right\rfloor\right)}\right\rangle\right|^{\alpha}\left(1-\mathrm{i} \tan \left(\frac{\pi \alpha}{2}\right) \operatorname{sign}\left(\left\langle n^{-1 / \alpha} \boldsymbol{\theta}_{n}, \boldsymbol{v}_{j}^{\left(\left\lfloor n t_{d}\right\rfloor\right)}\right\rangle\right)\right)\right\} \\
& \quad=\exp \left\{-C_{\alpha}\left(1-m_{\xi}^{\alpha}\right) \frac{1}{n} \sum_{j=0}^{\left\lfloor n t_{d}\right\rfloor}\left|\left\langle\boldsymbol{\theta}_{n}, \boldsymbol{v}_{j}^{\left(\left\lfloor n t_{d}\right\rfloor\right)}\right\rangle\right|^{\alpha}\left(1-\mathrm{i} \tan \left(\frac{\pi \alpha}{2}\right) \operatorname{sign}\left(\left\langle\boldsymbol{\theta}_{n}, \boldsymbol{v}_{j}^{\left(\left\lfloor n t_{d}\right\rfloor\right)}\right\rangle\right)\right)\right\} .
\end{aligned}
$$

We further have

$$
\begin{aligned}
\left\langle\boldsymbol{\theta}_{n}, \boldsymbol{v}_{0}^{\left(\left\lfloor n t_{d}\right\rfloor\right)}\right\rangle & =\sum_{i=1}^{d} \vartheta_{i} \sum_{k=\left\lfloor n t_{i-1}\right\rfloor+1}^{\left\lfloor n t_{i}\right\rfloor}\left\langle\boldsymbol{e}_{k+1}, \boldsymbol{v}_{0}^{\left(\left\lfloor n t_{d}\right\rfloor\right)}\right\rangle=\sum_{i=1}^{d} \vartheta_{i} \sum_{k=\left\lfloor n t_{i-1}\right\rfloor+1}^{\left\lfloor n t_{i}\right\rfloor} \frac{m_{\xi}^{k}}{\left(1-m_{\xi}^{\alpha}\right)^{1 / \alpha}} \\
& =\frac{1}{\left(1-m_{\xi}^{\alpha}\right)^{1 / \alpha}\left(1-m_{\xi}\right)} \sum_{i=1}^{d} \vartheta_{i}\left(m_{\xi}^{\left\lfloor n t_{i-1}\right\rfloor+1}-m_{\xi}^{\left\lfloor n t_{i}\right\rfloor+1}\right),
\end{aligned}
$$

and hence for each $\alpha \in(0,1) \cup(1,2)$,

$$
\frac{1}{n}\left|\left\langle\boldsymbol{\theta}_{n}, \boldsymbol{v}_{0}^{\left(\left\lfloor n t_{d}\right\rfloor\right)}\right\rangle\right|^{\alpha}\left(1-\mathrm{i} \tan \left(\frac{\pi \alpha}{2}\right) \operatorname{sign}\left(\left\langle\boldsymbol{\theta}_{n}, \boldsymbol{v}_{0}^{\left(\left\lfloor n t_{d}\right\rfloor\right)}\right\rangle\right)\right) \rightarrow 0 \quad \text { as } n \rightarrow \infty .
$$

The aim of the following discussion is showing that for all $\alpha \in(0,1) \cup(1,2)$ and $\ell \in\{1, \ldots, d\}$,

$$
\frac{1}{n} \sum_{j=\left\lfloor n t_{\ell-1}\right\rfloor+1}^{\left\lfloor n t_{\ell}\right\rfloor}\left|\left\langle\boldsymbol{\theta}_{n}, \boldsymbol{v}_{j}^{\left(\left\lfloor n t_{d}\right\rfloor\right)}\right\rangle\right|^{\alpha} \rightarrow \frac{\left(t_{\ell}-t_{\ell-1}\right)\left|\vartheta_{\ell}\right|^{\alpha}}{\left(1-m_{\xi}\right)^{\alpha}} \quad \text { as } n \rightarrow \infty .
$$


Here for all $\ell \in\{1, \ldots, d\}$ and $j \in\left\{\left\lfloor n t_{\ell-1}\right\rfloor+1, \ldots,\left\lfloor n t_{\ell}\right\rfloor\right\}$,

$$
\begin{aligned}
\left\langle\boldsymbol{\theta}_{n}, \boldsymbol{v}_{j}^{\left(\left\lfloor n t_{d}\right\rfloor\right)}\right\rangle & =\sum_{i=1}^{d} \vartheta_{i} \sum_{k=\left\lfloor n t_{i-1}\right\rfloor+1}^{\left\lfloor n t_{i}\right\rfloor}\left\langle\boldsymbol{e}_{k+1}, \boldsymbol{v}_{j}^{\left(\left\lfloor n t_{d}\right\rfloor\right)}\right\rangle=\sum_{i=1}^{d} \vartheta_{i} \sum_{k=\left\lfloor n t_{i-1}\right\rfloor+1}^{\left\lfloor n t_{i}\right\rfloor} m_{\xi}^{k-j} \mathbf{1}_{\{k \geqslant j\}} \\
& =\frac{1}{1-m_{\xi}}\left(\vartheta_{\ell}\left(1-m_{\xi}^{\left\lfloor n t_{\ell}\right\rfloor-j+1}\right)+\sum_{i=\ell+1}^{d} \vartheta_{i}\left(m_{\xi}^{\left\lfloor n t_{i-1}\right\rfloor-j+1}-m_{\xi}^{\left\lfloor n t_{i}\right\rfloor-j+1}\right)\right) .
\end{aligned}
$$

In case of $\alpha \in(0,1]$, we have

$$
|x|^{\alpha}-|y|^{\alpha} \leqslant|x+y|^{\alpha} \leqslant|x|^{\alpha}+|y|^{\alpha}, \quad x, y \in \mathbb{R} .
$$

In case of $\alpha \in(1,2)$, by the mean value theorem and (3.13) we have

$$
|| x+\left.y\right|^{\alpha}-|x|^{\alpha}|\leqslant \alpha| y\left|\max \left\{|x+y|^{\alpha-1},|x|^{\alpha-1}\right\} \leqslant \alpha\right| y \mid\left(|x|^{\alpha-1}+|y|^{\alpha-1}\right), \quad x, y \in \mathbb{R} .
$$

Hence for all $\alpha \in(0,2)$ and $x, y \in \mathbb{R}$, we obtain

$$
|x|^{\alpha}-2|y|\left(|x|^{\alpha-1}+|y|^{\alpha-1}\right) \leqslant|x+y|^{\alpha} \leqslant|x|^{\alpha}+2|y|\left(|x|^{\alpha-1}+|y|^{\alpha-1}\right),
$$

so, by (3.12) and the squeeze theorem, to prove (3.11), it suffices to check that

$$
\begin{aligned}
& \frac{1}{n} \sum_{j=\left\lfloor n t_{\ell-1}\right\rfloor+1}^{\left\lfloor n t_{\ell}\right\rfloor}\left(1-m_{\xi}^{\left\lfloor n t_{\ell}\right\rfloor-j+1}\right)^{\alpha} \rightarrow t_{\ell}-t_{\ell-1}, \\
& \frac{1}{n} \sum_{j=\left\lfloor n t_{\ell-1}\right\rfloor+1}^{\left\lfloor n t_{\ell}\right\rfloor}\left|\sum_{i=\ell+1}^{d} \vartheta_{i}\left(m_{\xi}^{\left\lfloor n t_{i-1}\right\rfloor-j+1}-m_{\xi}^{\left\lfloor n t_{i}\right\rfloor-j+1}\right)\right|^{\alpha} \rightarrow 0, \\
& \frac{1}{n} \sum_{j=\left\lfloor n t_{\ell-1}\right\rfloor+1}^{\left\lfloor n t_{\ell}\right\rfloor}\left|\sum_{i=\ell+1}^{d} \vartheta_{i}\left(m_{\xi}^{\left\lfloor n t_{i-1}\right\rfloor-j+1}-m_{\xi}^{\left\lfloor n t_{i}\right\rfloor-j+1}\right)\right|\left(1-m_{\xi}^{\left\lfloor n t_{\ell}\right\rfloor-j+1}\right)^{\alpha-1} \rightarrow 0
\end{aligned}
$$

as $n \rightarrow \infty$. Since $(1-t)^{\alpha}=1-\alpha t+o(t)$ as $t \downarrow 0$, there exists $j_{0} \in \mathbb{N}$ such that $\left|\left(1-m_{\xi}^{j}\right)^{\alpha}-1+\alpha m_{\xi}^{j}\right| \leqslant m_{\xi}^{j}$ for all $j \geqslant j_{0}$. Hence

$$
\begin{aligned}
\mid \frac{1}{n} & \sum_{j=\left\lfloor n t_{\ell-1}\right\rfloor+1}^{\left\lfloor n t_{\ell}\right\rfloor}\left(1-m_{\xi}^{\left\lfloor n t_{\ell}\right\rfloor-j+1}\right)^{\alpha}-\frac{1}{n} \sum_{j=1}^{\left\lfloor n t_{\ell}\right\rfloor-\left\lfloor n t_{\ell-1}\right\rfloor}\left(1-\alpha m_{\xi}^{j}\right) \mid \\
= & \left|\frac{1}{n} \sum_{j=1}^{\left\lfloor n t_{\ell}\right\rfloor-\left\lfloor n t_{\ell-1}\right\rfloor}\left(1-m_{\xi}^{j}\right)^{\alpha}-\frac{1}{n} \sum_{j=1}^{\left\lfloor n t_{\ell}\right\rfloor-\left\lfloor n t_{\ell-1}\right\rfloor}\left(1-\alpha m_{\xi}^{j}\right)\right| \\
& \leqslant\left|\frac{1}{n} \sum_{j=1}^{j_{0}-1}\left(\left(1-m_{\xi}^{j}\right)^{\alpha}-1+\alpha m_{\xi}^{j}\right)\right|+\frac{1}{n} \sum_{j=j_{0}}^{\left\lfloor n t_{\ell}\right\rfloor-\left\lfloor n t_{\ell-1}\right\rfloor} m_{\xi}^{j} \\
& \leqslant\left|\frac{1}{n} \sum_{j=1}^{j_{0}-1}\left(\left(1-m_{\xi}^{j}\right)^{\alpha}-1+\alpha m_{\xi}^{j}\right)\right|+\frac{1}{n} \frac{m_{\xi}^{j_{0}}}{1-m_{\xi}} \rightarrow 0 \text { as } n \rightarrow \infty .
\end{aligned}
$$


Thus

$$
\begin{aligned}
& \lim _{n \rightarrow \infty} \frac{1}{n} \sum_{j=\left\lfloor n t_{\ell-1}\right\rfloor+1}^{\left\lfloor n t_{\ell}\right\rfloor}\left(1-m_{\xi}^{\left\lfloor n t_{\ell}\right\rfloor-j+1}\right)^{\alpha} \\
& \quad=\lim _{n \rightarrow \infty} \frac{1}{n} \sum_{j=1}^{\left\lfloor n t_{\ell}\right\rfloor-\left\lfloor n t_{\ell-1}\right\rfloor}\left(1-\alpha m_{\xi}^{j}\right)=\lim _{n \rightarrow \infty} \frac{1}{n}\left(\left\lfloor n t_{\ell}\right\rfloor-\left\lfloor n t_{\ell-1}\right\rfloor-\alpha \frac{m_{\xi}-m_{\xi}^{\left\lfloor n t_{\ell}\right\rfloor-\left\lfloor n t_{\ell-1}\right\rfloor+1}}{1-m_{\xi}}\right) \\
& =t_{\ell}-t_{\ell-1},
\end{aligned}
$$

yielding (3.14). In case of $\alpha \in(1,2)$, for all $x_{1}, \ldots, x_{k} \in \mathbb{R}$, we have $\left|x_{1}+\cdots+x_{k}\right|^{\alpha} \leqslant k^{\alpha-1}\left(\left|x_{1}\right|^{\alpha}+\cdots+\right.$ $\left.\left|x_{k}\right|^{\alpha}\right)$, and hence by (3.13) for each $\alpha \in(0,2)$, we obtain

$$
\left|x_{1}+\cdots+x_{k}\right|^{\alpha} \leqslant k\left(\left|x_{1}\right|^{\alpha}+\cdots+\left|x_{k}\right|^{\alpha}\right), \quad x_{1}, \ldots, x_{k} \in \mathbb{R}
$$

Consequently, we have

$$
\begin{aligned}
& \frac{1}{n} \sum_{j=\left\lfloor n t_{\ell-1}\right\rfloor+1}^{\left\lfloor n t_{\ell}\right\rfloor}\left|\sum_{i=\ell+1}^{d} \vartheta_{i}\left(m_{\xi}^{\left\lfloor n t_{i-1}\right\rfloor-j+1}-m_{\xi}^{\left\lfloor n t_{i}\right\rfloor-j+1}\right)\right|^{\alpha} \\
& \quad \leqslant \frac{1}{n} \sum_{j=\left\lfloor n t_{\ell-1}\right\rfloor+1}^{\left\lfloor n t_{\ell}\right\rfloor}(d-\ell) \sum_{i=\ell+1}^{d}\left|\vartheta_{i}\right|^{\alpha}\left(m_{\xi}^{\left\lfloor n t_{i-1}\right\rfloor-j+1}-m_{\xi}^{\left\lfloor n t_{i}\right\rfloor-j+1}\right)^{\alpha} \\
& \quad \leqslant \frac{d}{n} \sum_{i=\ell+1}^{d}\left|\vartheta_{i}\right|^{\alpha} \sum_{j=\left\lfloor n t_{\ell-1}\right\rfloor+1}^{\left\lfloor n t_{\ell}\right\rfloor} m_{\xi}^{\left(\left\lfloor n t_{i-1}\right\rfloor-j+1\right) \alpha} \leqslant \frac{d}{n} \sum_{i=\ell+1}^{d}\left|\vartheta_{i}\right|^{\alpha} \sum_{k=0}^{\infty} m_{\xi}^{k \alpha} \rightarrow 0 \quad \text { as } n \rightarrow \infty,
\end{aligned}
$$

yielding (3.15). For each $n \in \mathbb{N}$ and for each $j \in\left\{\left\lfloor n t_{\ell-1}\right\rfloor+1, \ldots,\left\lfloor n t_{\ell}\right\rfloor\right\}$, we have

$$
\left(1-m_{\xi}^{\left\lfloor n t_{\ell}\right\rfloor-j+1}\right)^{\alpha-1} \leqslant \begin{cases}\left(1-m_{\xi}\right)^{\alpha-1} & \text { if } \alpha \in(0,1], \\ 1 & \text { if } \alpha \in(1,2),\end{cases}
$$

and hence

$$
\begin{aligned}
& \frac{1}{n} \sum_{j=\left\lfloor n t_{\ell-1}\right\rfloor+1}^{\left\lfloor n t_{\ell}\right\rfloor}\left|\sum_{i=\ell+1}^{d} \vartheta_{i}\left(m_{\xi}^{\left\lfloor n t_{i-1}\right\rfloor-j+1}-m_{\xi}^{\left\lfloor n t_{i}\right\rfloor-j+1}\right)\right|\left(1-m_{\xi}^{\left\lfloor n t_{\ell}\right\rfloor-j+1}\right)^{\alpha-1} \\
& \quad \leqslant \frac{\left(1-m_{\xi}\right)^{\alpha-1} \vee 1}{n} \sum_{j=\left\lfloor n t_{\ell-1}\right\rfloor+1}^{\left\lfloor n t_{\ell}\right\rfloor} \sum_{i=\ell+1}^{d}\left|\vartheta_{i}\right|\left(m_{\xi}^{\left\lfloor n t_{i-1}\right\rfloor-j+1}-m_{\xi}^{\left\lfloor n t_{i}\right\rfloor-j+1}\right) \\
& \quad \leqslant \frac{\left(1-m_{\xi}\right)^{\alpha-1} \vee 1}{n} \sum_{i=\ell+1}^{d}\left|\vartheta_{i}\right| \sum_{j=\left\lfloor n t_{\ell-1}\right\rfloor+1}^{\left\lfloor n t_{\ell}\right\rfloor} m_{\xi}^{\left\lfloor n t_{i-1}\right\rfloor-j+1} \\
& \quad \leqslant \frac{\left(1-m_{\xi}\right)^{\alpha-1} \vee 1}{n} \sum_{i=\ell+1}^{d}\left|\vartheta_{i}\right| \sum_{k=0}^{\infty} m_{\xi}^{k} \rightarrow 0 \quad \text { as } n \rightarrow \infty,
\end{aligned}
$$

yielding (3.16). Thus we obtain (3.11). 
Next, we show that for each $\ell \in\{1, \ldots, d\}$,

$$
\frac{1}{n} \sum_{j=\left\lfloor n t_{\ell-1}\right\rfloor+1}^{\left\lfloor n t_{\ell}\right\rfloor}\left|\left\langle\boldsymbol{\theta}_{n}, \boldsymbol{v}_{j}^{\left(\left\lfloor n t_{d}\right\rfloor\right)}\right\rangle\right|^{\alpha} \operatorname{sign}\left(\left\langle\boldsymbol{\theta}_{n}, \boldsymbol{v}_{j}^{\left(\left\lfloor n t_{d}\right\rfloor\right)}\right\rangle\right) \rightarrow \frac{\left(t_{\ell}-t_{\ell-1}\right)\left|\vartheta_{\ell}\right|^{\alpha}}{\left(1-m_{\xi}\right)^{\alpha}} \operatorname{sign}\left(\vartheta_{\ell}\right) \quad \text { as } n \rightarrow \infty .
$$

If $\vartheta_{\ell}=0$, then this readily follows from (3.12) and (3.15). If $\vartheta_{\ell} \neq 0$, then we show that there exists $\widetilde{C}_{\ell} \in \mathbb{R}_{++}$ such that

$$
\operatorname{sign}\left(\left\langle\boldsymbol{\theta}_{n}, \boldsymbol{v}_{j}^{\left(\left\lfloor n t_{d}\right\rfloor\right)}\right\rangle\right)=\operatorname{sign}\left(\vartheta_{\ell}\right)
$$

for each $n \in \mathbb{N}$ and each $j \in\left\{\left\lfloor n t_{\ell-1}\right\rfloor+1, \ldots,\left\lfloor n t_{\ell}\right\rfloor\right\}$ with $j<\left\lfloor n t_{\ell}\right\rfloor+1-\widetilde{C}_{\ell}$. First, observe that, by (3.12), the inequality

$$
\left|\sum_{i=\ell+1}^{d} \vartheta_{i}\left(m_{\xi}^{\left\lfloor n t_{i-1}\right\rfloor-j+1}-m_{\xi}^{\left\lfloor n t_{i}\right\rfloor-j+1}\right)\right|<\left|\vartheta_{\ell}\left(1-m_{\xi}^{\left\lfloor n t_{\ell}\right\rfloor-j+1}\right)\right|
$$

implies (3.17). Then we have

$$
\begin{aligned}
& \left|\sum_{i=\ell+1}^{d} \vartheta_{i}\left(m_{\xi}^{\left\lfloor n t_{i-1}\right\rfloor-j+1}-m_{\xi}^{\left\lfloor n t_{i}\right\rfloor-j+1}\right)\right| \\
& \quad \leqslant\left(\max _{\ell+1 \leqslant i \leqslant d}\left|\vartheta_{i}\right|\right) \sum_{i=\ell+1}^{d}\left(m_{\xi}^{\left\lfloor n t_{i-1}\right\rfloor-j+1}-m_{\xi}^{\left\lfloor n t_{i}\right\rfloor-j+1}\right) \\
& \quad=\sum_{i=\ell+1}^{d}\left|\vartheta_{i}\right|\left(m_{\xi}^{\left\lfloor n t_{\ell}\right\rfloor-j+1}-m_{\xi}^{\left\lfloor n t_{d}\right\rfloor-j+1}\right) \leqslant \sum_{i=\ell+1}^{d}\left|\vartheta_{i}\right| m_{\xi}^{\left\lfloor n t_{\ell}\right\rfloor-j+1},
\end{aligned}
$$

and hence (3.18) is satisfied if

$$
\sum_{i=\ell+1}^{d}\left|\vartheta_{i}\right| m_{\xi}^{\left\lfloor n t_{\ell}\right\rfloor-j+1}<\left|\vartheta_{\ell}\right|\left(1-m_{\xi}^{\left\lfloor n t_{\ell}\right\rfloor-j+1}\right)
$$

which is satisfied if $m_{\xi}^{\left\lfloor n t_{\ell}\right\rfloor-j+1}<\left|\vartheta_{\ell}\right| /\left(\left|\vartheta_{\ell}\right|+\cdots+\left|\vartheta_{d}\right|\right)$ or, equivalently, if

$$
j<\left\lfloor n t_{\ell}\right\rfloor+1-\widetilde{C}_{\ell} \quad \text { with } \widetilde{C}_{\ell}:=\frac{\log \left(\frac{\left|\vartheta_{\ell}\right|}{\left|\vartheta_{\ell}\right|+\cdots+\left|\vartheta_{d}\right|}\right)}{\log \left(m_{\xi}\right)} \in \mathbb{R}_{++} .
$$

Hence, for $\vartheta_{\ell} \neq 0, n \in \mathbb{N}$, and $j \in\left\{\left\lfloor n t_{\ell-1}\right\rfloor+1, \ldots,\left\lfloor n t_{\ell}\right\rfloor\right\}$ with $j<\left\lfloor n t_{\ell}\right\rfloor+1-\widetilde{C}_{\ell}$, we have (3.17). Moreover, for all $n \in \mathbb{N}$ and $j \in\left\{\left\lfloor n t_{\ell-1}\right\rfloor+1, \ldots,\left\lfloor n t_{\ell}\right\rfloor\right\}$, by (3.12) we have

$$
\begin{aligned}
\left|\left\langle\boldsymbol{\theta}_{n}, \boldsymbol{v}_{j}^{\left(\left\lfloor n t_{d}\right\rfloor\right)}\right\rangle\right| & \leqslant \frac{1}{1-m_{\xi}}\left(\left|\vartheta_{\ell}\right|\left(1-m_{\xi}^{\left\lfloor n t_{\ell}\right\rfloor-j+1}\right)+\sum_{i=\ell+1}^{d}\left|\vartheta_{i}\right|\left(m_{\xi}^{\left\lfloor n t_{i-1}\right\rfloor-j+1}-m_{\xi}^{\left\lfloor n t_{i}\right\rfloor-j+1}\right)\right) \\
& \leqslant \frac{1}{1-m_{\xi}} \sum_{i=\ell}^{d}\left|\vartheta_{i}\right|,
\end{aligned}
$$


yielding that

$$
\begin{aligned}
& \left.\left|\frac{1}{n} \sum_{j=\left\lfloor n t_{\ell}\right\rfloor+1-\widetilde{C}_{\ell}}^{\left\lfloor n t_{\ell}\right\rfloor}\right|\left\langle\boldsymbol{\theta}_{n}, \boldsymbol{v}_{j}^{\left(\left\lfloor n t_{d}\right\rfloor\right)}\right\rangle\right|^{\alpha} \operatorname{sign}\left(\left\langle\boldsymbol{\theta}_{n}, \boldsymbol{v}_{j}^{\left(\left\lfloor n t_{d}\right\rfloor\right)}\right\rangle\right) \mid \\
& \quad \leqslant \frac{1}{n} \sum_{j=\left\lfloor n t_{\ell}\right\rfloor+1-\widetilde{C}_{\ell}}^{\left\lfloor n t_{\ell}\right\rfloor}\left|\left\langle\boldsymbol{\theta}_{n}, \boldsymbol{v}_{j}^{\left(\left\lfloor n t_{d}\right\rfloor\right)}\right\rangle\right|^{\alpha} \leqslant \frac{\widetilde{C}_{\ell}}{n\left(1-m_{\xi}\right)^{\alpha}}\left(\sum_{i=\ell}^{d}\left|\vartheta_{i}\right|\right)^{\alpha} \rightarrow 0 \quad \text { as } n \rightarrow \infty .
\end{aligned}
$$

Consequently, by (3.11) we have

$$
\begin{aligned}
& \lim _{n \rightarrow \infty} \frac{1}{n} \sum_{j=\left\lfloor n t_{\ell-1}\right\rfloor+1}^{\left\lfloor n t_{\ell}\right\rfloor}\left|\left\langle\boldsymbol{\theta}_{n}, \boldsymbol{v}_{j}^{\left(\left\lfloor n t_{d}\right\rfloor\right)}\right\rangle\right|^{\alpha} \operatorname{sign}\left(\left\langle\boldsymbol{\theta}_{n}, \boldsymbol{v}_{j}^{\left(\left\lfloor n t_{d}\right\rfloor\right)}\right\rangle\right) \\
& =\lim _{n \rightarrow \infty} \frac{1}{n} \sum_{\left\lfloor n t_{\ell-1}\right\rfloor+1 \leqslant j<\left\lfloor n t_{\ell}\right\rfloor+1-\widetilde{C}_{\ell}}\left|\left\langle\boldsymbol{\theta}_{n}, \boldsymbol{v}_{j}^{\left(\left\lfloor n t_{d}\right\rfloor\right)}\right\rangle\right|^{\alpha} \operatorname{sign}\left(\left\langle\boldsymbol{\theta}_{n}, \boldsymbol{v}_{j}^{\left(\left\lfloor n t_{d}\right\rfloor\right)}\right\rangle\right) \\
& =\lim _{n \rightarrow \infty} \frac{1}{n} \sum_{\left\lfloor n t_{\ell-1}\right\rfloor+1 \leqslant j<\left\lfloor n t_{\ell}\right\rfloor+1-\widetilde{C}_{\ell}}\left|\left\langle\boldsymbol{\theta}_{n}, \boldsymbol{v}_{j}^{\left(\left\lfloor n t_{d}\right\rfloor\right)}\right\rangle\right|^{\alpha} \operatorname{sign}\left(\vartheta_{\ell}\right) \\
& \quad=\lim _{n \rightarrow \infty} \frac{1}{n} \sum_{j=\left\lfloor n t_{\ell-1}\right\rfloor+1}^{\left\lfloor n t_{\ell}\right\rfloor}\left|\left\langle\boldsymbol{\theta}_{n}, \boldsymbol{v}_{j}^{\left(\left\lfloor n t_{d}\right\rfloor\right)}\right\rangle\right|^{\alpha} \operatorname{sign}\left(\vartheta_{\ell}\right)=\frac{\left(t_{\ell}-t_{\ell-1}\right)\left|\vartheta_{\ell}\right|^{\alpha}}{\left(1-m_{\xi}\right)^{\alpha}} \operatorname{sign}\left(\vartheta_{\ell}\right),
\end{aligned}
$$

as desired. We conclude that for all $\alpha \in(0,1) \cup(1,2)$,

$$
\begin{aligned}
& \mathbf{E}\left(\exp \left\{\mathrm{i} \sum_{\ell=1}^{d} \frac{\vartheta_{\ell}}{n^{1 / \alpha}} \sum_{k=\left\lfloor n t_{\ell-1}\right\rfloor+1}^{\left\lfloor n t_{\ell}\right\rfloor}\left(\mathcal{Y}_{k}^{(\alpha)}+\frac{\alpha}{1-\alpha}\right)\right\}\right) \\
& =\mathbf{E}\left(\exp \left\{\mathrm{i}\left\langle n^{-1 / \alpha} \boldsymbol{\theta}_{n}, \boldsymbol{\mathcal { X }}_{1}^{\left(\left\lfloor n t_{d}\right\rfloor, \alpha\right)}+\frac{\alpha}{1-\alpha} \mathbf{1}_{\left\lfloor n t_{d}\right\rfloor+1}\right\rangle\right\}\right) \\
& \quad \rightarrow \exp \left\{-C_{\alpha} \frac{1-m_{\xi}^{\alpha}}{\left(1-m_{\xi}\right)^{\alpha}} \sum_{\ell=1}^{d}\left(t_{\ell}-t_{\ell-1}\right)\left|\vartheta_{\ell}\right|^{\alpha}\left(1-\mathrm{i} \tan \left(\frac{\pi \alpha}{2}\right) \operatorname{sign}\left(\vartheta_{\ell}\right)\right)\right\} \\
& =\mathbf{E}\left(\exp \left\{\mathrm{i} \sum_{\ell=1}^{d} \vartheta_{\ell}\left(\left(\mathcal{Z}_{t_{\ell}}^{(\alpha)}+\frac{\alpha}{1-\alpha} t_{\ell}\right)-\left(\mathcal{Z}_{t_{\ell-1}}^{(\alpha)}+\frac{\alpha}{1-\alpha} t_{\ell-1}\right)\right)\right\}\right) \text { as } n \rightarrow \infty .
\end{aligned}
$$

By the continuity theorem we obtain that for all $\alpha \in(0,1) \cup(1,2)$,

$$
\begin{aligned}
& \left(\frac{1}{n^{1 / \alpha}} \sum_{k=\left\lfloor n t_{\ell-1}\right\rfloor+1}^{\left\lfloor n t_{\ell}\right\rfloor}\left(\mathcal{Y}_{k}^{(\alpha)}+\frac{\alpha}{1-\alpha}\right)\right)_{\ell \in\{1, \ldots, d\}} \\
& \quad \stackrel{\mathcal{D}}{\longrightarrow}\left(\left(\mathcal{Z}_{t_{\ell}}^{(\alpha)}+\frac{\alpha}{1-\alpha} t_{\ell}\right)-\left(\mathcal{Z}_{t_{\ell-1}}^{(\alpha)}+\frac{\alpha}{1-\alpha} t_{\ell-1}\right)\right)_{\ell \in\{1, \ldots, d\}}
\end{aligned}
$$

as $n \rightarrow \infty$, and hence the continuous mapping theorem yields (3.10), and we have finished the proofs of (2.3), (2.4), and (2.6). 
Now we turn to prove (2.5). For each $n \in \mathbb{N}$, by Corollary 2 and the continuous mapping theorem, in case of $\alpha=1$, we obtain

$$
\left(\frac{1}{n \log (n) a_{N}} \sum_{k=1}^{\lfloor n t\rfloor} \sum_{j=1}^{N}\left(X_{k}^{(j)}-\mathbf{E}\left(X_{k}^{(j)} \mathbf{1}_{\left\{X_{k}^{(j)} \leqslant a_{N}\right\}}\right)\right)\right)_{t \in \mathbb{R}_{+}} \stackrel{\mathcal{D}_{\mathrm{f}}}{\longrightarrow}\left(\frac{1}{n \log (n)} \sum_{k=1}^{\lfloor n t\rfloor} \mathcal{Y}_{k}^{(1)}\right)_{t \in \mathbb{R}_{+}} \quad \text { as } N \rightarrow \infty .
$$

Consequently, to prove (2.5), we need to show that

$$
\left(\frac{1}{n \log (n)} \sum_{k=1}^{\lfloor n t\rfloor} \mathcal{Y}_{k}^{(1)}\right)_{t \in \mathbb{R}_{+}} \stackrel{\mathcal{D}_{\mathrm{f}}}{\longrightarrow}(t)_{t \in \mathbb{R}_{+}} \text {as } n \rightarrow \infty .
$$

Since the limit in (3.19) is deterministic, by van der Vaart [28, Thm. 2.7, part (vi)] it suffices to show that for each $t \in \mathbb{R}_{+}$, we have

$$
\frac{1}{n \log (n)} \sum_{k=1}^{\lfloor n t\rfloor} \mathcal{Y}_{k}^{(1)} \stackrel{\mathcal{D}}{\longrightarrow} t \quad \text { as } n \rightarrow \infty
$$

For all $n \in \mathbb{N}, t \in \mathbb{R}_{+}$, and $\vartheta \in \mathbb{R}$, we have

$$
\mathbf{E}\left(\exp \left\{\mathrm{i} \frac{\vartheta}{n \log (n)} \sum_{k=1}^{\lfloor n t\rfloor} \mathcal{Y}_{k}^{(1)}\right\}\right)=\mathbf{E}\left(\exp \left\{\mathrm{i}\left\langle\frac{\vartheta \mathbf{1}_{\lfloor n t\rfloor+1}}{n \log (n)}, \boldsymbol{\mathcal { X }}_{1}^{(\lfloor n t\rfloor, 1)}\right\rangle\right\}\right)
$$

By the explicit form of the characteristic function of $\mathcal{X}_{1}^{(\lfloor n t\rfloor, 1)}$ given in Theorem 1,

$$
\begin{aligned}
& \mathbf{E}\left(\exp \left\{\mathrm{i}\left\langle\frac{\vartheta \mathbf{1}_{\lfloor n t\rfloor+1}}{n \log (n)}, \boldsymbol{\mathcal { X }}_{1}^{(\lfloor n t\rfloor, 1)}\right\rangle\right\}\right) \\
& =\exp \left\{-\frac{C_{1}\left(1-m_{\xi}\right)|\vartheta|}{n \log (n)} \sum_{j=0}^{\lfloor n t\rfloor}\left\langle\mathbf{1}_{\lfloor n t\rfloor+1}, \boldsymbol{v}_{j}^{(\lfloor n t\rfloor)}\right\rangle\left(1+\mathrm{i} \frac{2}{\pi} \operatorname{sign}(\vartheta) \log \left(\frac{|\vartheta|}{n \log (n)}\left\langle\mathbf{1}_{\lfloor n t\rfloor+1}, \boldsymbol{v}_{j}^{(\lfloor n t\rfloor)}\right\rangle\right)\right)\right. \\
& \left.\quad+\mathrm{i} C \frac{\vartheta}{n \log (n)}\left\langle\mathbf{1}_{\lfloor n t\rfloor+1}, \mathbf{1}_{\lfloor n t\rfloor+1}\right\rangle+\mathrm{i} \frac{\left(1-m_{\xi}\right) \vartheta}{n \log (n)} \sum_{j=0}^{\lfloor n t\rfloor} \sum_{\ell=j+1}^{\lfloor n t\rfloor+1}\left\langle\boldsymbol{e}_{\ell}, \boldsymbol{v}_{j}^{(\lfloor n t\rfloor)}\right\rangle \log \left(\left\langle\boldsymbol{e}_{\ell}, \boldsymbol{v}_{j}^{(\lfloor n t\rfloor)}\right\rangle\right)\right\} \\
& \rightarrow \mathrm{e}^{\mathrm{i} t \vartheta}
\end{aligned}
$$

as $n \rightarrow \infty$ for each $\vartheta \in \mathbb{R}$. Indeed,

$$
\begin{gathered}
\frac{1}{n \log (n)}\left\langle\mathbf{1}_{\lfloor n t\rfloor+1}, \mathbf{1}_{\lfloor n t\rfloor+1}\right\rangle=\frac{\lfloor n t\rfloor+1}{n \log (n)} \rightarrow 0 \quad \text { as } n \rightarrow \infty, \\
\left|\frac{1}{n \log (n)} \sum_{j=0}^{\lfloor n t\rfloor} \sum_{\ell=j+1}^{\lfloor n t\rfloor+1}\left\langle\boldsymbol{e}_{\ell}, \boldsymbol{v}_{j}^{(\lfloor n t\rfloor)}\right\rangle \log \left(\left\langle\boldsymbol{e}_{\ell}, \boldsymbol{v}_{j}^{(\lfloor n t\rfloor)}\right\rangle\right)\right| \\
=\left|\frac{1}{n \log (n)} \sum_{j=0}^{\lfloor n t\rfloor} \sum_{\ell=j+1}^{\lfloor n t\rfloor+1} m_{\xi}^{\ell-j-1} \log \left(m_{\xi}^{\ell-j-1}\right)\right| \leqslant \frac{\left|\log \left(m_{\xi}\right)\right|}{n \log (n)} \sum_{j=0}^{\lfloor n t\rfloor} \sum_{\ell=j+1}^{\lfloor n t\rfloor+1}(\ell-j-1) m_{\xi}^{\ell-j-1}
\end{gathered}
$$




$$
\begin{aligned}
& \leqslant \frac{\left|\log \left(m_{\xi}\right)\right|}{n \log (n)} \sum_{j=0}^{\lfloor n t\rfloor} \sum_{\ell=j+1}^{\infty}(\ell-j-1) m_{\xi}^{\ell-j-1}=\frac{\left|\log \left(m_{\xi}\right)\right|(\lfloor n t\rfloor+1)}{n \log (n)} \sum_{k=0}^{\infty} k m_{\xi}^{k} \\
& =\frac{m_{\xi}\left|\log \left(m_{\xi}\right)\right|(\lfloor n t\rfloor+1)}{\left(1-m_{\xi}\right)^{2} n \log (n)} \rightarrow 0 \quad \text { as } n \rightarrow \infty, \\
& \frac{1}{n \log (n)} \sum_{j=0}^{\lfloor n t\rfloor}\left\langle\mathbf{1}_{\lfloor n t\rfloor+1}, \boldsymbol{v}_{j}^{(\lfloor n t\rfloor)}\right\rangle \\
& =\frac{1}{n \log (n)} \sum_{j=0}^{\lfloor n t\rfloor} \frac{1-m_{\xi}^{\lfloor n t\rfloor-j+1}}{1-m_{\xi}} \leqslant \frac{\lfloor n t\rfloor+1}{\left(1-m_{\xi}\right) n \log (n)} \rightarrow 0 \quad \text { as } n \rightarrow \infty,
\end{aligned}
$$

and

$$
\begin{aligned}
& -\frac{C_{1}\left(1-m_{\xi}\right)|\vartheta|}{n \log (n)} \sum_{j=0}^{\lfloor n t\rfloor}\left\langle\mathbf{1}_{\lfloor n t\rfloor+1}, \boldsymbol{v}_{j}^{(\lfloor n t\rfloor)}\right\rangle \mathrm{i} \frac{2}{\pi} \operatorname{sign}(\vartheta) \log \left(\frac{|\vartheta|}{n \log (n)}\left\langle\mathbf{1}_{\lfloor n t\rfloor+1}, \boldsymbol{v}_{j}^{(\lfloor n t\rfloor)}\right\rangle\right) \\
& \rightarrow \mathrm{i} t \vartheta \quad \text { as } n \rightarrow \infty,
\end{aligned}
$$

since

$$
\begin{aligned}
& \left|\log \left(\frac{|\vartheta|}{\log (n)}\left\langle\mathbf{1}_{\lfloor n t\rfloor+1}, \boldsymbol{v}_{j}^{(\lfloor n t\rfloor)}\right\rangle\right)\right| \\
& \quad=\left|\log \left(\frac{|\vartheta|}{\log (n)} \frac{1-m_{\xi}^{\lfloor n t\rfloor-j+1}}{1-m_{\xi}}\right)\right|=\left|\log (|\vartheta|)-\log (\log (n))+\log \left(\frac{1-m_{\xi}^{\lfloor n t\rfloor-j+1}}{1-m_{\xi}}\right)\right| \\
& \quad \leqslant|\log (|\vartheta|)|+\log (\log (n))+\left|\log \left(1-m_{\xi}\right)\right|,
\end{aligned}
$$

hence by (3.21)

$$
\begin{aligned}
& \left|\frac{1}{n \log (n)} \sum_{j=0}^{\lfloor n t\rfloor}\left\langle\mathbf{1}_{\lfloor n t\rfloor+1}, \boldsymbol{v}_{j}^{(\lfloor n t\rfloor)}\right\rangle \log \left(\frac{|\vartheta|}{\log (n)}\left\langle\mathbf{1}_{\lfloor n t\rfloor+1}, \boldsymbol{v}_{j}^{(\lfloor n t\rfloor)}\right\rangle\right)\right| \\
& \quad \leqslant \frac{\lfloor n t\rfloor+1}{\left(1-m_{\xi}\right) n \log (n)}\left(|\log (|\vartheta|)|+\log (\log (n))+\left|\log \left(1-m_{\xi}\right)\right|\right) \rightarrow 0 \quad \text { as } n \rightarrow \infty,
\end{aligned}
$$

and

$$
\begin{aligned}
& \frac{C_{1}\left(1-m_{\xi}\right)|\vartheta|}{n \log (n)} \sum_{j=0}^{\lfloor n t\rfloor}\left\langle\mathbf{1}_{\lfloor n t\rfloor+1}, \boldsymbol{v}_{j}^{(\lfloor n t\rfloor)}\right\rangle \mathrm{i} \frac{2}{\pi} \operatorname{sign}(\vartheta) \log (n) \\
& \quad=\mathrm{i} \frac{\left(1-m_{\xi}\right) \vartheta}{n} \sum_{j=0}^{\lfloor n t\rfloor} \frac{1-m_{\xi}^{\lfloor n t\rfloor-j+1}}{1-m_{\xi}} \rightarrow \mathrm{i} t \vartheta \quad \text { as } n \rightarrow \infty .
\end{aligned}
$$

By the continuity theorem we obtain (3.20), and hence we have finished the proof of (2.5). 


\section{Appendix A: The underlying space and vague convergence}

For each $d \in \mathbb{N}$, put $\mathbb{R}_{0}^{d}:=\mathbb{R}^{d} \backslash\{\mathbf{0}\}$, and denote by $\mathcal{B}\left(\mathbb{R}_{0}^{d}\right)$ the Borel $\sigma$-algebra of $\mathbb{R}_{0}^{d}$ induced by the metric $\varrho: \mathbb{R}_{0}^{d} \times \mathbb{R}_{0}^{d} \rightarrow \mathbb{R}_{+}$given by

$$
\varrho(\boldsymbol{x}, \boldsymbol{y}):=\min \{\|\boldsymbol{x}-\boldsymbol{y}\|, 1\}+\left|\frac{1}{\|\boldsymbol{x}\|}-\frac{1}{\|\boldsymbol{y}\|}\right|, \quad \boldsymbol{x}, \boldsymbol{y} \in \mathbb{R}_{0}^{d} .
$$

Lemma A1. The set $\mathbb{R}_{0}^{d}$ equipped with the metric $\varrho$ given in (A.1) is a complete separable metric space, and $B \subset \mathbb{R}_{0}^{d}$ is bounded with respect to the metric $\varrho$ if and only if $B$ is separated from the origin $\mathbf{0} \in \mathbb{R}^{d}$, that is, there exists $\varepsilon \in \mathbb{R}_{++}$such that $B \subset\left\{\boldsymbol{x} \in \mathbb{R}_{0}^{d}:\|\boldsymbol{x}\|>\varepsilon\right\}$. Moreover, the topology and the Borel $\sigma$-algebra $\mathcal{B}\left(\mathbb{R}_{0}^{d}\right)$ on $\mathbb{R}_{0}^{d}$ induced by the metric @ coincides with the topology and the Borel $\sigma$-algebra on $\mathbb{R}_{0}^{d}$ induced by the usual metric $d(\boldsymbol{x}, \boldsymbol{y}):=\|\boldsymbol{x}-\boldsymbol{y}\|, \boldsymbol{x}, \boldsymbol{y} \in \mathbb{R}_{0}^{d}$, respectively.

For the proof of Lemma A1, see our arXiv preprint Barczy et al. [4].

Since $\mathbb{R}_{0}^{d}$ is locally compact, second countable, and Hausdorff, we can choose a metric such that the relatively compact sets are precisely the bounded ones; see Kallenberg [15, p. 18]. The metric $\varrho$ does not have this property, but we do not need it.

Write $\left(\mathbb{R}_{0}^{d}\right)^{\curlyvee}$ for the class of bounded Borel sets with respect to the metric $\varrho$ given in (A.1). A measure $\nu$ on $\left(\mathbb{R}_{0}^{d}, \mathcal{B}\left(\mathbb{R}_{0}^{d}\right)\right)$ is said to be locally finite if $\nu(B)<\infty$ for every $B \in\left(\mathbb{R}_{0}^{d}\right)^{\curlyvee}$ and write $\mathcal{M}\left(\mathbb{R}_{0}^{d}\right)$ for the class of locally finite measures on $\left(\mathbb{R}_{0}^{d}, \mathcal{B}\left(\mathbb{R}_{0}^{d}\right)\right)$.

Write $\widehat{C}_{\mathbb{R}_{0}^{d}}$ for the class of bounded continuous functions $f: \mathbb{R}_{0}^{d} \rightarrow \mathbb{R}_{+}$with bounded support. Hence, if $f \in \widehat{C}_{\mathbb{R}_{0}^{d}}$, then there exists $\varepsilon \in \mathbb{R}_{++}$such that $f(\boldsymbol{x})=0$ for all $\boldsymbol{x} \in \mathbb{R}_{0}^{d}$ with $\|\boldsymbol{x}\| \leqslant \varepsilon$. The vague topology on $\mathcal{M}\left(\mathbb{R}_{0}^{d}\right)$ is constructed as in Kallenberg [15, Chap. 4]. The associated notion of vague convergence of a sequence $\left(\nu_{n}\right)_{n \in \mathbb{N}}$ in $\mathcal{M}\left(\mathbb{R}_{0}^{d}\right)$ toward $\nu \in \mathcal{M}\left(\mathbb{R}_{0}^{d}\right)$, denoted by $\nu_{n} \stackrel{v}{\rightarrow} \nu$ as $n \rightarrow \infty$, is defined by the condition $\nu_{n}(f) \rightarrow \nu(f)$ as $n \rightarrow \infty$ for all $f \in \widehat{C}_{\mathbb{R}_{0}^{d}}$, where $\kappa(f):=\int_{\mathbb{R}_{0}^{d}} f(\boldsymbol{x}) \kappa(\mathrm{d} \boldsymbol{x})$ for $\kappa \in \mathcal{M}\left(\mathbb{R}_{0}^{d}\right)$.

If $\nu$ is a measure on $\left(\mathbb{R}_{0}^{d}, \mathcal{B}\left(\mathbb{R}_{0}^{d}\right)\right)$, then $B \in \mathcal{B}\left(\mathbb{R}_{0}^{d}\right)$ is called a $\nu$-continuity set if $\nu(\partial B)=0$, and the class of bounded $\nu$-continuity sets is denoted by $\left(\mathbb{R}_{0}^{d}\right)_{\nu}$. The following statement is an analogue of the portmanteau theorem for vague convergence; see, for example, Kallenberg [14, 15.7.2].

Lemma A2. Let $\nu, \nu_{n} \in \mathcal{M}\left(\mathbb{R}_{0}^{d}\right), n \in \mathbb{N}$. Then the following statements are equivalent:

(i) $\nu_{n} \stackrel{v}{\rightarrow} \nu$ as $n \rightarrow \infty$,

(ii) $\nu_{n}(B) \rightarrow \nu(B)$ as $n \rightarrow \infty$ for all $B \in\left(\mathbb{R}_{0}^{d}\right)_{\nu}$.

\section{Appendix B: Regularly varying distributions}

First, we recall the notions of slowly varying and regularly varying functions, respectively.

DEFinition B1. A measurable function $U: \mathbb{R}_{++} \rightarrow \mathbb{R}_{++}$is called regularly varying at infinity with index $\rho \in \mathbb{R}$ if for all $c \in \mathbb{R}_{++}$,

$$
\lim _{x \rightarrow \infty} \frac{U(c x)}{U(x)}=c^{\rho}
$$

In case of $\rho=0$, we call $U$ slowly varying at infinity.

Definition B2. A random variable $Y$ is called regularly varying with index $\alpha \in \mathbb{R}_{++}$if $\mathbf{P}(|Y|>x) \in \mathbb{R}_{++}$ for all $x \in \mathbb{R}_{++}$, the function $\mathbb{R}_{++} \ni x \mapsto \mathbf{P}(|Y|>x) \in \mathbb{R}_{++}$is regularly varying at infinity with index $-\alpha$, and the following tail-balance condition holds:

$$
\lim _{x \rightarrow \infty} \frac{\mathbf{P}(Y>x)}{\mathbf{P}(|Y|>x)}=p, \quad \lim _{x \rightarrow \infty} \frac{\mathbf{P}(Y \leqslant-x)}{\mathbf{P}(|Y|>x)}=q, \quad \text { where } p+q=1 .
$$


Remark B1. In the tail-balance condition (B.1) the second convergence can be replaced by

$$
\lim _{x \rightarrow \infty} \frac{\mathbf{P}(Y<-x)}{\mathbf{P}(|Y|>x)}=q
$$

see our arXiv preprint Barczy et al. [4].

\section{Lemma B1.}

(i) A nonnegative random variable $Y$ is regularly varying with index $\alpha \in \mathbb{R}_{++}$if and only if $\mathbf{P}(Y>x) \in$ $\mathbb{R}_{++}$for all $x \in \mathbb{R}_{++}$and the function $\mathbb{R}_{++} \ni x \mapsto \mathbf{P}(Y>x) \in \mathbb{R}_{++}$is regularly varying at infinity with index $-\alpha$.

(ii) If $Y$ is a regularly varying random variable with index $\alpha \in \mathbb{R}_{++}$, then for each $\beta \in \mathbb{R}_{++},|Y|^{\beta}$ is regularly varying with index $\alpha / \beta$.

Lemma B2. If $Y$ is a regularly varying random variable with index $\alpha \in \mathbb{R}_{++}$, then there exists a sequence $\left(a_{n}\right)_{n \in \mathbb{N}}$ in $\mathbb{R}_{++}$such that $n \mathbf{P}\left(|Y|>a_{n}\right) \rightarrow 1$ as $n \rightarrow \infty$. If $\left(a_{n}\right)_{n \in \mathbb{N}}$ is such a sequence, then $a_{n} \rightarrow \infty$ as $n \rightarrow \infty$.

Lemma B3 [Karamata's theorem for truncated moments]. Consider a nonnegative regularly varying random variable $Y$ with index $\alpha \in \mathbb{R}_{++}$. Then

$$
\begin{aligned}
& \lim _{x \rightarrow \infty} \frac{x^{\beta} \mathbf{P}(Y>x)}{\mathbf{E}\left(Y^{\beta} \mathbf{1}_{\{Y \leqslant x\}}\right)}=\frac{\beta-\alpha}{\alpha} \quad \text { for } \beta \in[\alpha, \infty), \\
& \lim _{x \rightarrow \infty} \frac{x^{\beta} \mathbf{P}(Y>x)}{\mathbf{E}\left(Y^{\beta} \mathbf{1}_{\{Y>x\}}\right)}=\frac{\alpha-\beta}{\alpha} \quad \text { for } \beta \in(-\infty, \alpha) .
\end{aligned}
$$

For Lemma B3, see, for example, Bingham et al. [8, pp. 26-27] or Buraczewski et al. [9, Appendix B.4].

Next, based on Buraczewski et al. [9, Appendix C], we recall the definition and some properties of regularly varying random vectors.

DEFINITION B3. A $d$-dimensional random vector $\boldsymbol{Y}$ and its distribution are called regularly varying with index $\alpha \in \mathbb{R}_{++}$if there exists a probability measure $\psi$ on $\mathbb{S}^{d-1}$ such that for all $c \in \mathbb{R}_{++}$,

$$
\frac{\mathbf{P}\left(\|\boldsymbol{Y}\|>c x, \frac{\boldsymbol{Y}}{\|\boldsymbol{Y}\|} \in \cdot\right)}{\mathbf{P}(\|\boldsymbol{Y}\|>x)} \stackrel{w}{\longrightarrow} c^{-\alpha} \psi(\cdot) \quad \text { as } x \rightarrow \infty,
$$

where $\stackrel{w}{\rightarrow}$ denotes the weak convergence of finite measures on $\mathbb{S}^{d-1}$. The probability measure $\psi$ is called the spectral measure of $\boldsymbol{Y}$.

The following equivalent characterization of multivariate regular variation can be derived, for example, from Resnick [24, p. 69].

Proposition B1. A d-dimensional random vector $\boldsymbol{Y}$ is regularly varying with some index $\alpha \in \mathbb{R}_{++}$if and only if there exists a nonzero locally finite measure $\mu$ on $\mathbb{R}_{0}^{d}$ satisfying the limit relation

$$
\mu_{x}(\cdot):=\frac{\mathbf{P}\left(x^{-1} \boldsymbol{Y} \in \cdot\right)}{\mathbf{P}(\|\boldsymbol{Y}\|>x)} \stackrel{v}{\longrightarrow} \mu(\cdot) \quad \text { as } x \rightarrow \infty,
$$

where $\stackrel{v}{\rightarrow}$ denotes the vague convergence of locally finite measures on $\mathbb{R}_{0}^{d}$ (see Appendix A for the notion $\stackrel{v}{\rightarrow}$ ). Further, $\mu$ satisfies the property $\mu(c B)=c^{-\alpha} \mu(B)$ for any $c \in \mathbb{R}_{++}$and $B \in \mathcal{B}\left(\mathbb{R}_{0}^{d}\right)$ (see, e.g., Theorems 1.14 and 1.15 and Remark 1.16 in Lindskog [16]). 
The measure $\mu$ in Proposition B1 is called the limit measure of $\boldsymbol{Y}$. For the proof of Proposition B1, see our arXiv preprint Barczy et al. [4].

The next statement follows, for example, from part (i) in Lemma C.3.1 in Buraczewski et al. [9].

Lemma B4. If $\boldsymbol{Y}$ is a regularly varying d-dimensional random vector with index $\alpha \in \mathbb{R}_{++}$, then for each $\boldsymbol{c} \in \mathbb{R}^{d}$, the random vector $\boldsymbol{Y}-\boldsymbol{c}$ is regularly varying with index $\alpha$.

Recall that if $\boldsymbol{Y}$ is a regularly varying $d$-dimensional random vector with index $\alpha \in \mathbb{R}_{++}$and limit measure $\mu$ given in (B.2), and $f: \mathbb{R}^{d} \rightarrow \mathbb{R}$ is a continuous function with $f^{-1}(\{0\})=\{\mathbf{0}\}$ that is positively homogeneous of degree $\beta \in \mathbb{R}_{++}$(i.e., $f(c \boldsymbol{v})=c^{\beta} f(\boldsymbol{v})$ for every $c \in \mathbb{R}_{++}$and $\boldsymbol{v} \in \mathbb{R}^{d}$ ), then $f(\boldsymbol{Y})$ is regularly varying with index $\alpha / \beta$ and limit measure $\mu\left(f^{-1}(\cdot)\right)$; see, for example, Buraczewski et al. [9, p. 282]. Next we describe the tail behavior of $f(\boldsymbol{Y})$ for appropriate positively homogeneous functions $f: \mathbb{R}^{d} \rightarrow \mathbb{R}$.

Proposition B2. Let $\boldsymbol{Y}$ be a regularly varying d-dimensional random vector with index $\alpha \in \mathbb{R}_{++}$, and let $f: \mathbb{R}^{d} \rightarrow \mathbb{R}$ be a measurable function that is positively homogeneous of degree $\beta \in \mathbb{R}_{++}$, continuous at $\mathbf{0}$ and such that $\mu\left(D_{f}\right)=0$, where $\mu$ is the limit measure of $\boldsymbol{Y}$ given in (B.2), and $D_{f}$ denotes the set of discontinuities of $f$. Then $\mu\left(\partial_{\mathbb{R}_{0}^{d}}\left(f^{-1}((1, \infty))\right)\right)=0$, where $\partial_{\mathbb{R}_{0}^{d}}\left(f^{-1}((1, \infty))\right)$ denotes the boundary of $f^{-1}((1, \infty))$ in $\mathbb{R}_{0}^{d}$. Consequently,

$$
\lim _{x \rightarrow \infty} \frac{\mathbf{P}(f(\boldsymbol{Y})>x)}{\mathbf{P}\left(\|\boldsymbol{Y}\|^{\beta}>x\right)}=\mu\left(f^{-1}((1, \infty))\right)
$$

and $f(\boldsymbol{Y})$ is regularly varying with tail index $\alpha / \beta$.

\section{Appendix C: Weak convergence of partial-sum processes toward Lévy processes}

We formulate a slight modification of Theorem 7.1 in Resnick [25] with a different centering.

Theorem C1. Suppose that for each $N \in \mathbb{N}, \boldsymbol{X}_{N, j}, j \in \mathbb{N}$, are independent identically distributed d-dimensional random vectors such that

$$
N \mathbf{P}\left(\boldsymbol{X}_{N, 1} \in \cdot\right) \stackrel{v}{\longrightarrow} \nu(\cdot) \quad \text { on } \mathbb{R}_{0}^{d} \text { as } N \rightarrow \infty
$$

where $\nu$ is a Lévy measure on $\mathbb{R}_{0}^{d}$ such that $\nu\left(\left\{\boldsymbol{x} \in \mathbb{R}_{0}^{d}:\left|\left\langle\boldsymbol{e}_{\ell}, \boldsymbol{x}\right\rangle\right|=1\right\}\right)=0$ for every $\ell \in\{1, \ldots, d\}$, and that

$$
\lim _{\varepsilon \downarrow 0} \limsup _{N \rightarrow \infty} N \mathbf{E}\left(\left\langle\boldsymbol{e}_{\ell}, \boldsymbol{X}_{N, 1}\right\rangle^{2} \mathbf{1}_{\left\{\left|\left\langle\boldsymbol{e}_{\ell}, \boldsymbol{X}_{N, 1}\right\rangle\right| \leqslant \varepsilon\right\}}\right)=0, \quad \ell \in\{1, \ldots, d\} .
$$

Then we have

$$
\left(\sum_{j=1}^{\lfloor N t\rfloor}\left(\boldsymbol{X}_{N, j}-\sum_{\ell=1}^{d} \mathbf{E}\left(\left\langle\boldsymbol{e}_{\ell}, \boldsymbol{X}_{N, j}\right\rangle \mathbf{1}_{\left\{\left|\left\langle\boldsymbol{e}_{\ell}, \boldsymbol{X}_{N, j}\right\rangle\right| \leqslant 1\right\}}\right) \boldsymbol{e}_{\ell}\right)\right)_{t \in \mathbb{R}_{+}} \stackrel{\mathcal{D}}{\longrightarrow}\left(\boldsymbol{\mathcal { X }}_{t}\right)_{t \in \mathbb{R}_{+}} \text {as } N \rightarrow \infty,
$$

where $\left(\mathcal{X}_{t}\right)_{t \in \mathbb{R}_{+}}$is a Lévy process such that the characteristic function of the distribution $\mu$ of $\mathcal{X}_{1}$ has the form

$$
\widehat{\mu}(\boldsymbol{\theta})=\exp \left\{\int_{\mathbb{R}_{0}^{d}}\left(\mathrm{e}^{\mathrm{i}\langle\boldsymbol{\theta}, \boldsymbol{x}\rangle}-1-\mathrm{i} \sum_{\ell=1}^{d}\left\langle\boldsymbol{e}_{\ell}, \boldsymbol{\theta}\right\rangle\left\langle\boldsymbol{e}_{\ell}, \boldsymbol{x}\right\rangle \mathbf{1}_{(0,1]}\left(\left|\left\langle\boldsymbol{e}_{\ell}, \boldsymbol{x}\right\rangle\right|\right)\right) \nu(\mathrm{d} \boldsymbol{x})\right\}, \quad \boldsymbol{\theta} \in \mathbb{R}^{d} .
$$

Proof. See our arXiv preprint Barczy et al. [4]. 


\section{Appendix D: Tail behavior of $\left(X_{k}\right)_{k \in \mathbb{Z}_{+}}$}

Due to Basrak et al. [6, Thm. 2.1.1], we have the following tail behavior.

Theorem D1. We have

$$
\lim _{x \rightarrow \infty} \frac{\pi((x, \infty))}{\mathbf{P}(\varepsilon>x)}=\sum_{i=0}^{\infty} m_{\xi}^{i \alpha}=\frac{1}{1-m_{\xi}^{\alpha}},
$$

where $\pi$ denotes the unique stationary distribution of the Markov chain $\left(X_{k}\right)_{k \in \mathbb{Z}_{+}}$, and, consequently, $\pi$ is also regularly varying with index $\alpha$.

Note that in case of $\alpha=1$ and $m_{\varepsilon}=\infty$, Basrak et al. [6, Thm. 2.1.1] additionally assume that $\varepsilon$ is consistently varying (or, in other words, intermediate varying), but, eventually, it follows from the fact that $\varepsilon$ is regularly varying.

Let $\left(X_{k}\right)_{k \in \mathbb{Z}}$ be a strongly stationary extension of $\left(X_{k}\right)_{k \in \mathbb{Z}_{+}}$. Basrak et al. [6, Lemma 3.1] described the socalled forward tail process of the strongly stationary process $\left(X_{k}\right)_{k \in \mathbb{Z}}$, and hence, due to Basrak and Segers [7, Thm. 2.1], the strongly stationary process $\left(X_{k}\right)_{k \in \mathbb{Z}}$ is jointly regularly varying.

Theorem D2. The finite-dimensional conditional distributions of $\left(x^{-1} X_{k}\right)_{k \in \mathbb{Z}_{+}}$with respect to the condition $X_{0}>x$ converge weakly to the corresponding finite-dimensional distributions of $\left(m_{\xi}^{k} Y\right)_{k \in \mathbb{Z}_{+}}$as $x \rightarrow \infty$, where $Y$ is a random variable with Pareto distribution $\mathbf{P}(Y \leqslant y)=\left(1-y^{-\alpha}\right) \mathbf{1}_{[1, \infty)}(y), y \in \mathbb{R}$. Consequently, the strongly stationary process $\left(X_{k}\right)_{k \in \mathbb{Z}}$ is jointly regularly varying with index $\alpha$, that is, all its finite-dimensional distributions are regularly varying with index $\alpha$. The process $\left(m_{\xi}^{k} Y\right)_{k \in \mathbb{Z}_{+}}$is the so-called

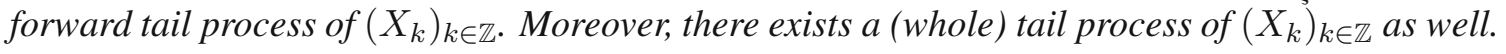

By the proof of Theorem 1 and Proposition D1 we obtain the following results.

Proposition D1. For each $k \in \mathbb{Z}_{+}$,

(i) the limit measure $\widetilde{\nu}_{k, \alpha}$ of $\left(X_{0}, \ldots, X_{k}\right)^{\top}$ given in (3.3) takes the form

$$
\widetilde{\nu}_{k, \alpha}=\frac{\nu_{k, \alpha}}{\nu_{k, \alpha}\left(\left\{\boldsymbol{x} \in \mathbb{R}_{0}^{k+1}:\|\boldsymbol{x}\|>1\right\}\right)},
$$

where $\nu_{k, \alpha}$ is given by (3.4), and

$$
\nu_{k, \alpha}\left(\left\{\boldsymbol{x} \in \mathbb{R}_{0}^{k+1}:\|\boldsymbol{x}\|>1\right\}\right)=\frac{1-m_{\xi}^{\alpha}}{\left(1-m_{\xi}^{2}\right)^{\alpha / 2}}\left(\frac{\left(1-m_{\xi}^{2(k+1)}\right)^{\alpha / 2}}{1-m_{\xi}^{\alpha}}+\sum_{j=1}^{k}\left(1-m_{\xi}^{2(k-j+1)}\right)^{\alpha / 2}\right)
$$

(ii) the tail behavior of $X_{0}+\cdots+X_{k}$ is given by

$$
\lim _{x \rightarrow \infty} \frac{\mathbf{P}\left(X_{0}+\cdots+X_{k}>x\right)}{\mathbf{P}\left(X_{0}>x\right)}=\frac{1-m_{\xi}^{\alpha}}{\left(1-m_{\xi}\right)^{\alpha}}\left(\frac{\left(1-m_{\xi}^{k+1}\right)^{\alpha}}{1-m_{\xi}^{\alpha}}+\sum_{j=1}^{k}\left(1-m_{\xi}^{k-j+1}\right)^{\alpha}\right) .
$$

Proof. (i) In the proof of Theorem 1, we derived $\nu_{k, \alpha}=\widetilde{\nu}_{k, \alpha} / \widetilde{\nu}_{k, \alpha}\left(\left\{\boldsymbol{x} \in \mathbb{R}_{0}^{k+1}: x_{0}>1\right\}\right)$. Consequently,

$$
\widetilde{\nu}_{k, \alpha}\left(\left\{\boldsymbol{x} \in \mathbb{R}_{0}^{k+1}: x_{0}>1\right\}\right)=\frac{\widetilde{\nu}_{k, \alpha}\left(\left\{\boldsymbol{x} \in \mathbb{R}_{0}^{k+1}:\|\boldsymbol{x}\|>1\right\}\right)}{\nu_{k, \alpha}\left(\left\{\boldsymbol{x} \in \mathbb{R}_{0}^{k+1}:\|\boldsymbol{x}\|>1\right\}\right)},
$$


where using Proposition D1 with the 1-homogeneous function $\mathbb{R}^{k+1} \ni \boldsymbol{x} \mapsto\|\boldsymbol{x}\|$, we have

$$
\widetilde{\nu}_{k, \alpha}\left(\left\{\boldsymbol{x} \in \mathbb{R}_{0}^{k+1}:\|\boldsymbol{x}\|>1\right\}\right)=\lim _{x \rightarrow \infty} \frac{\mathbf{P}\left(\left\|\left(X_{0}, \ldots, X_{k}\right)^{\top}\right\|>x\right)}{\mathbf{P}\left(\left\|\left(X_{0}, \ldots, X_{k}\right)^{\top}\right\|>x\right)}=1,
$$

and, by (3.4),

$$
\begin{aligned}
\nu_{k, \alpha} & \left(\left\{\boldsymbol{x} \in \mathbb{R}_{0}^{k+1}:\|\boldsymbol{x}\|>1\right\}\right) \\
& =\int_{\mathbb{R}_{0}^{k+1}} \mathbf{1}_{\{\|\boldsymbol{x}\|>1\}} \nu_{k, \alpha}(\mathrm{d} \boldsymbol{x})=\left(1-m_{\xi}^{\alpha}\right) \sum_{j=0}^{k} \int_{0}^{\infty} \mathbf{1}_{\left\{\left\|u \boldsymbol{v}_{j}^{(k)}\right\|>1\right\}} \alpha u^{-\alpha-1} \mathrm{~d} u \\
& =\left(1-m_{\xi}^{\alpha}\right) \sum_{j=0}^{k} \int_{\left\|\boldsymbol{v}_{j}^{(k)}\right\|^{-1}}^{\infty} \alpha u^{-\alpha-1} \mathrm{~d} u=\left(1-m_{\xi}^{\alpha}\right) \sum_{j=0}^{k}\left\|\boldsymbol{v}_{j}^{(k)}\right\|^{\alpha} \\
& =\left(1-m_{\xi}^{\alpha}\right)\left(\frac{\left(1+m_{\xi}^{2}+\cdots+m_{\xi}^{2 k}\right)^{\alpha / 2}}{1-m_{\xi}^{\alpha}}+\sum_{j=1}^{k}\left(1+m_{\xi}^{2}+\cdots+m_{\xi}^{2(k-j)}\right)^{\alpha / 2}\right) \\
& =\frac{1-m_{\xi}^{\alpha}}{\left(1-m_{\xi}^{2}\right)^{\alpha / 2}}\left(\frac{\left(1-m_{\xi}^{2(k+1)}\right)^{\alpha / 2}}{1-m_{\xi}^{\alpha}}+\sum_{j=1}^{k}\left(1-m_{\xi}^{2(k-j+1)}\right)^{\alpha / 2}\right) .
\end{aligned}
$$

(ii) Applying Proposition D1 to the 1-homogeneous functions $\mathbb{R}^{k+1} \ni \boldsymbol{x} \mapsto x_{0}$ and $\mathbb{R}^{k+1} \ni \boldsymbol{x} \mapsto$ $x_{0}+\cdots+x_{k}$ and formula (3.4), we obtain

$$
\begin{aligned}
\lim _{x \rightarrow \infty} \frac{\mathbf{P}\left(X_{0}+\cdots+X_{k}>x\right)}{\mathbf{P}\left(X_{0}>x\right)} & =\lim _{x \rightarrow \infty} \frac{\mathbf{P}\left(\left\|\left(X_{0}, \ldots, X_{k}\right)^{\top}\right\|>x\right)}{\mathbf{P}\left(X_{0}>x\right)} \frac{\mathbf{P}\left(X_{0}+\cdots+X_{k}>x\right)}{\mathbf{P}\left(\left\|\left(X_{0}, \ldots, X_{k}\right)^{\top}\right\|>x\right)} \\
& =\frac{\widetilde{\nu}_{k, \alpha}\left(\left\{\boldsymbol{x} \in \mathbb{R}_{0}^{k+1}: x_{0}+\cdots+x_{k}>1\right\}\right)}{\widetilde{\nu}_{k, \alpha}\left(\left\{\boldsymbol{x} \in \mathbb{R}_{0}^{k+1}: x_{0}>1\right\}\right)} \\
& =\nu_{k, \alpha}\left(\left\{\boldsymbol{x} \in \mathbb{R}_{0}^{k+1}: x_{0}+\cdots+x_{k}>1\right\}\right),
\end{aligned}
$$

which yields the statement as in part (i).

\section{References}

1. A. Araujo and E. Giné, The Central Limit Theorem for Real and Banach Valued Random Variables, Wiley Ser. Probab. Math. Stat., John Wiley \& Sons, New York, Chichester, Brisbane, 1980.

2. M. Barczy, Zs. Bősze, and G. Pap, On tail behaviour of stationary second-order Galton-Watson processes with immigration, 2018, arXiv:1801.07931.

3. M. Barczy, F. K. Nedényi, and G. Pap, On aggregation of multitype Galton-Watson branching processes with immigration, Mod. Stoch., Theory Appl., 5(1):53-79, 2018, available from: https://doi.org/10.15559/ 18 -vmsta95.

4. M. Barczy, F. K. Nedényi, and G. Pap, On aggregation of subcritical Galton-Watson branching processes with regularly varying immigration, 2019, arXiv:1906.00373.

5. M. Barczy, F. Nedényi, and G. Pap, Iterated limits for aggregation of randomized INAR(1) processes with Poisson innovations, J. Math. Anal. Appl., 451(1):524-543, 2017, available from: https://doi.org/10.1016/j. jmaa.2017.02.031. 
6. B. Basrak, R. Kulik, and Z. Palmowski, Heavy-tailed branching process with immigration, Stoch. Models, 29(4):413434, 2013, available from: https://doi.org/10.1080/15326349.2013.838508.

7. B. Basrak and J. Segers, Regularly varying multivariate time series, Stochastic Processes Appl., 119(4):1055-1080, 2009, available from: https://doi.org/10.1016/j.spa.2008.05.004.

8. N.H. Bingham, C.M. Goldie, and J.L. Teugels, Regular Variation, Encycl. Math. Appl., Vol. 27, Cambridge Univ. Press, Cambridge, 1987, available from: https://doi.org/10.1017/CB09780511721434.

9. D. Buraczewski, E. Damek, and T. Mikosch, Stochastic Models With Power-Law Tails. The equation $X=A X+B$, Springer Ser. Oper. Res. Financ. Eng., Springer, Cham, 2016, available from: https://doi.org/10.1007/ https: //978-3-319-29679-1.

10. C.W.J. Granger, Long memory relationships and the aggregation of dynamic models, J. Econom., 14(2):227-238, 1980, available from: https://doi.org/10.1016/0304-4076(80) 90092-5.

11. J. Jacod and A.N. Shiryaev, Limit Theorems for Stochastic Processes, 2nd ed., Grundlehren Math. Wiss., Vol. 288, Springer, Berlin, 2003, available from: https://doi.org/10.1007/978-3-662-05265-5.

12. A. Janssen and J. Segers, Markov tail chains, J. Appl. Probab., 51(4):1133-1153, 2014, available from: https: //doi.org/10.1239/jap/1421763332.

13. M. Jirak, Limit theorems for aggregated linear processes, Adv. Appl. Probab., 45(2):520-544, 2013, available from: https://doi.org/10.1239/aap/1370870128.

14. O. Kallenberg, Random Measures,3rd ed., Akademie-Verlag/Academic Press, Berlin/London, 1983.

15. O. Kallenberg, Random Measures, Theory and Applications, Probab. Theory Stoch. Model., Vol. 77, Springer, Cham, 2017, available from: https://doi.org/10.1007/978-3-319-41598-7.

16. F. Lindskog, Multivariate Extremes and Regular Variation for Stochastic Processes, Diss. ETH No. 15319, Swiss Federal Institute of Technology Zürich, 2004, available from: https://doi.org/10.3929/ethz-a004669275 .

17. T. Mikosch and $\mathrm{O}$. Wintenberger, The cluster index of regularly varying sequences with applications to limit theory for functions of multivariate Markov chains, Probab. Theory Relat. Fields, 159(1-2):157-196, 2014, available from: https://doi.org/10.1007/s00440-013-0504-1.

18. V. Pilipauskaite, V. Skorniakov, and D. Surgailis, Joint temporal and contemporaneous aggregation of randomcoefficient AR(1) processes with infinite variance, Adv. Appl. Probab., 52(1):237-265, 2020, available from: https://doi.org/10.1017/apr.2019.59.

19. V. Pilipauskaite and D. Surgailis, Joint temporal and contemporaneous aggregation of random-coefficient $\operatorname{AR}(1)$ processes, Stochastic Processes Appl., 124(2):1011-1035,2014, available from: https : / / doi . org/10.1016/ j.spa.2013.10.004.

20. H. Planinić and P. Soulier, The tail process revisited, Extremes, 21(4):551-579, 2018, available from: https : //doi.org/10.1007/s10687-018-0312-1.

21. D. Puplinskaite and D. Surgailis, Aggregation of random-coefficient $\operatorname{ar}(1)$ process with infinite variance and common innovations, Lith. Math. J., 49(4):446-463, 2009, available from: https://doi.org/10.1007/ https://s10986-009-9060-x.

22. D. Puplinskaite and D. Surgailis, Aggregation of a random-coefficient AR(1) process with infinite variance and idiosyncratic innovations, Adv. Appl. Probab., 42(2):509-527, 2010, available from: https://doi.org/10. $1239 /$ aap/1275055240.

23. M.P. Quine, The multi-type Galton-Watson process with immigration, J. Appl. Probab., 7:411-422, 1970, available from: https://doi.org/10.1017/s0021900200034975.

24. S.I. Resnick, Point processes, regular variation and weak convergence, Adv. Appl. Probab., 18(1):66-138, 1986, available from: https://doi.org/10.2307/1427239. 
25. S.I. Resnick, Heavy-Tail Phenomena, Springer Ser. Oper. Res. Financ. Eng., Springer, New York, 2007, available from: https://doi.org/10.1007/978-0-387-45024-7.

26. P.M. Robinson, Statistical inference for a random coefficient autoregressive model, Scand. J. Stat., 5(3):163-168, 1978, available from: http: //www. jstor.org/stable/pdf/4615707.pdf.

27. K.-i. Sato, Lévy Processes and Infinitely Divisible Distributions, Camb. Stud. Adv. Math., Vol 68, Cambridge Univ. Press, Cambridge, 1999. Translated from the 1990 Japanese original. Revised by the author.

28. A.W. Van der Vaart, Asymptotic Statistics, Camb. Ser. Stat. Probab. Math., Vol. 3, Cambridge Univ. Press, Cambridge, 1998, available from: https://doi.org/10.1017/CBO9780511802256.

29. N.M. Yanev, V.K. Stoimenova, and D.V. Atanasov, Stochastic modeling and estimation of COVID-19 population dynamics, C. R. Acad. Bulg. Sci., 73(4):451-460,2020, available from: https : / / doi . org/10 . $7546 /$ CRABS . 2020.04 .02 . 